\title{
Efficient 3D Analysis Of Laminate Structures Using ABD-Equivalent Material Models
}

\author{
Goldy Kumar ${ }^{\mathrm{a}, *}$, Vadim Shapiro $^{\mathrm{a}}$ \\ ${ }^{a}$ Spatial Automation Lab, Mechanical Engineering, University of Wisconsin- Madison, USA
}

\begin{abstract}
Laminate composites are widely used in automotive, aerospace, and increasingly in consumer industries, due to their reduced weight and superior structural properties. However, structural analysis of complex laminate structures remains challenging. 2D finite element methods based on plate/shell theories may be accurate and efficient, but they generally do not apply to the whole structure and require identification and preprocessing of the regions where the underlying assumptions hold. Fully automated structural analysis using solid 3D elements with sufficiently high order basis functions is possible in principle, but is rarely practiced due to the significant increase in the cost of computational integration over a large number of laminate plies.

We propose a procedure to replace the original laminate by much simpler new virtual material models. These virtual material models, under the usual assumptions made in lamination theory, have the same constitutive relationship as the corresponding 2D plate model of the original laminate, but require only a small fraction of computational integration costs in 3D FEA. We describe implementation of 3D FEA using these material models in a meshfree system using second order B-spline basis functions. Finally, we demonstrate their validity by showing agreement between computed and known results for standard problems.
\end{abstract}

Keywords: Composite, Laminate, Finite Element Method, Material Model

\section{Introduction}

\subsection{Motivation}

Laminate composites are now used widely in variety of industries, including aerospace, automobile, medical and sports $[1,2,3]$. Laminates are lightweight and stiff with customizable material properties, resulting in structures superior to those made of homogeneous materials $[4,3,2]$. High stiffness-to-weight ratio is achieved using fiber reinforced plies. These plies, when fused together under high temperature and pressure, form complex monolithic laminate parts. The fiber reinforcements, laid using techniques ranging from manual

${ }^{*}$ Corresponding author. Phone +1-608-334-9646

Email addresses: gkumar3@wisc.edu (Goldy Kumar), vshapiro@engr.wisc.edu (Vadim Shapiro) 


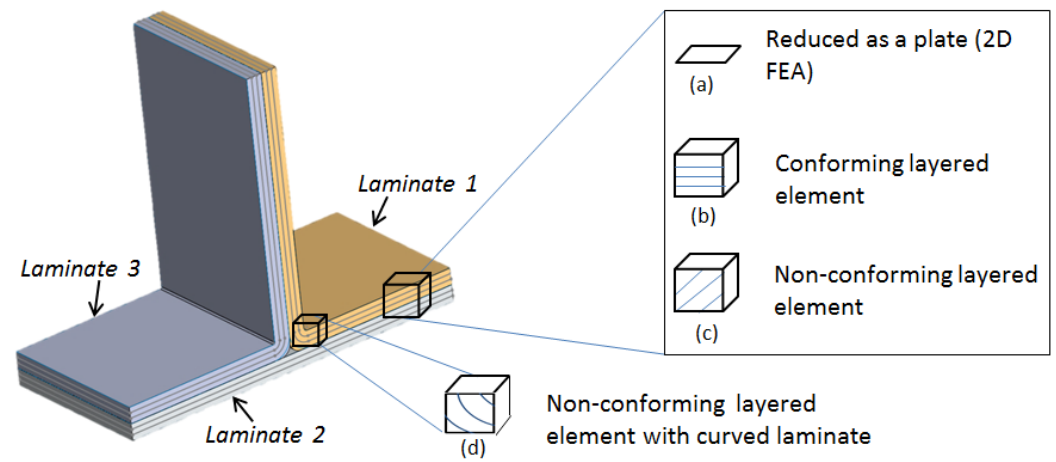

Figure 1: Figure shows a structure made of 3 laminates analyzed using different finite element methods (a) Plate/shell element (b) Element of 3D conforming mesh (c) Element of a 3D non-conforming mesh (d) Element of a non-conforming mesh with curved laminate inside.

to fully automatic, are generally parallel and unidirectional and, therefore, result in plies which are anisotropic in nature. Material properties are customized by varying fiber angle within each ply, controlling the number of plies, and adding additional materials between plies such as cores and fillers. The presence of numerous plies, however, leads to complex geometry and material distribution in laminate structures, and, therefore, structural analysis of laminates by treating each ply layer individually is prohibitively expensive. The common practice is to assume that the layers are permanently fused together and ignore any fluctuation in stress-strain fields at the interfaces of layers $[4,3,5,6]$. These assumptions allow to approximate laminate's global behavior as that of a plate or a shell. Interlaminar stresses and strains may be significant in boundary regions and regions of discontinuities [7] where full three-dimensional and/or layered methods should be used, but the plate/shell assumptions give sufficiently accurate stress and strain estimates for regions away from those regions[8]. In this paper, we will show that the same plate/shell assumptions, when applicable, may be used within a general 3D finite element analysis to dramatically speed up the analysis procedure.

Structural analysis of laminates can be carried out using different finite element methods, and some of them are illustrated for a typical laminate part in Figure 1. During finite element analysis (FEA), stiffness matrix $\mathbf{K}_{e}$ for each element must be computed, which in general form is given as [9]:

$$
\mathbf{K}_{e}=\int_{\Omega_{e}} \mathbf{B}^{T} \cdot \mathbf{Q} \cdot \mathbf{B} d \Omega
$$

where $\mathbf{B}$ is the strain-displacement matrix, $\mathbf{Q}$ is the material constitutive relation matrix, and $\Omega_{e}$ is the element's domain over which integration is done. Since there are numerous plies, meshing each ply independently requires a large number of elements and is, therefore, prohibitively expensive. A much smaller number of elements is needed if layered element method [10, 11, 12] is used (Figure 1b and 1c), wherein multiple plies can cross through an element. Such finite element methods (also called 3D FEA since integration domain $\Omega_{e}$ is $3 \mathrm{D}$ ) may be further classified into conforming and non-conforming (or meshfree), 
depending on whether the finite element mesh conforms to the geometry of the laminate. Three dimensional elements may exhibit locking and ill-conditioning of stiffness matrix when used for laminates that are thin in the ply layup direction $[9,13]$. These problems can be alleviated or eliminated by using higher order hierarchical ${ }^{1}$ basis functions $[14,15]$.

All 3D FEA methods are computationally expensive, as the above integration has to be carried over large number of plies (tens or even hundreds). Integration is performed using quadrature rules that depend on the geometry of the element as well as the degree of the integrand, and amounts to sampling the integrand at a number of quadrature points [9]. To get an idea of the high cost of integration for laminates, we consider the layered element used in reference [10] to analyze a laminate made of 100 plies. The element used is an eight-node brick element with tri-linear basis functions, which, for a homogeneous material, is fully integrated using 2 integration points in each direction, or 8 integration points in total [10]. However, in a laminate, 8 integration points are needed for each ply, which results in a 100 fold increase for our 100-ply laminate. Since integration cost represents a significant portion of the overall solution procedure, analysis of composite laminates using layered elements is an expensive proposition.

Plate or shell assumptions reduce the computation cost and increase accuracy of FEA for laminates owing to their thin walled nature. These assumptions may lead to different lamination theories, where the material matrices $\mathbf{Q}$ of all the plies are replaced by the so-called ABD matrices $[4,16]$. The structure and the integration domain $\Omega_{e}$ effectively reduces to a surface (Figure 1a), which is why this method is also called 2D FEA. However, 2D FEA is not valid in regions near boundaries and discontinuities (Figure 1), which have significant 3D stresses and, therefore, plate/shell assumptions are invalid. In this sense, 2D FEA methods are not general, because such regions are common in laminate structures.

\subsection{Contributions and Outline}

Based on the above discussion, the choice between 2D and 3D FEA amounts to a tradeoff between generality and computational efficiency. We seek to develop an approach to analysis of composite laminate structures that is as general as 3D FEA and as efficient as 2D FEA when dimensional reduction makes sense.

Specifically, we propose a method to reduce the excessive cost of integration for layered elements by taking advantage of plate/shell nature of laminates, whenever such assumptions are reasonable. To this end, we have devised a procedure to obtain material models which are simpler but are equivalent to the original laminate, under the assumption made in lamination theories. We refer to these new material models as $A B D$-equivalent material models, as they result in the same ABD matrices as the original laminate and, therefore, can replace the original laminate during integration if plate/shell assumptions apply. We demonstrate the effectiveness of two such material models - a 3-ply and a graded material model - in a non-conforming FEA system using layered solid elements. We validate the two ABD-equivalent material models by using them to analyze several benchmark problems,

\footnotetext{
${ }^{1} \mathrm{~A}$ basis function is called hierarchical when a higher order basis function contains all the lower order basis functions; for example, B-splines are hierarchical basis functions.
} 


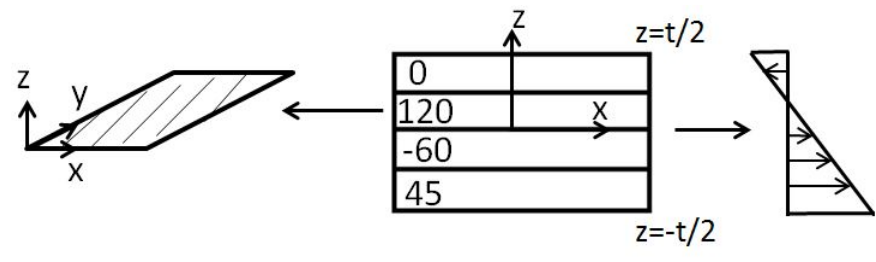

(A)

(B)

(C)

Figure 2: A) Ply with fiber angle $120^{\circ}$ B) $x z$ cross section of a laminate with plies at angles $45 /-60 / 120 / 0$ and C) Linear variation of strain $\epsilon_{x}$ along $z$ while laminate is in stretching and pure bending

and compare obtained results from known results. The fully implemented non-conforming FEA system uses layered solid elements with second-degree B-spline basis functions that are hierarchical in nature.

A brief outline of the paper is as follows. In section 2, we survey the related work. Section 3 develops the concept of ABD-equivalent material models and proposes two specific examples of such models. Implementation of the proposed approach in combination with a non-conforming finite element method is described in Section 4. Its effectiveness is demonstrated using a number of benchmark problems in Section 5, followed by conclusions and future work in Section 6.

\section{Related Work}

The finite element methods for simulating global behavior of laminate structures $[16,17$, 18] may be broadly classified as a $2 \mathrm{D}$ or a 3D FEA. For the purposes of this paper, we only consider those methods which ignore inter-ply phenomena, but we note that the inter-layer stress-strain can be partially predicted from global deformation $[4,19]$.

\subsection{Two-dimensional FEA}

Laminates usually behave as plates or shells, and are analyzed using 2D FEA. Depending on the strain field assumed in the laminate's thickness direction, different lamination theories exist [5, 17, 16], and can be classified as one of the following: Classical Lamination Plate Theory (CLPT), First Order Shear Deformation Theory (FSDT), or Higher Order Shear Deformation Theories (HSDTs). CLPT assumes laminates undergo only stretching and pure bending (Figure 2C): in-plane strains vary linearly in the thickness direction, and outof-plane strains are absent. On the other hand, FSTD and HSDT, as the names suggest, assume non-zero out-of-plane shear strains; FSDT assumes constant variation while HSDTs assume linear or even higher order [19] variation for out-of-plane shear strains. In-plane strains are assumed to be identical to CLPT, and out-of-plane normal strain is absent. Since our goal in this paper is to show that ABD-Equivalent material models can be used to simplify the actual material and, therefore, reduce the cost of 3D FEA, we only focus on CLPT and FSDT and not higher order theories. Figure 2 illustrates a laminate and the variation of strain field in $x$ direction along the thickness obtained using CLPT. 


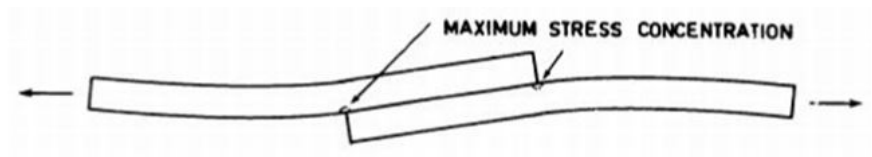

(A)

(B)

Figure 3: A) A lap joint bending under in-plane loading, which leads to high stress concentration near the joint $[20,21]$ B) When analyzed as a 2D structure, bending in lap joint is not captured at all.

As discussed above, although 2D FEA is efficient, it is not general. In addition, 2D FEA suffers from additional drawbacks that limit its applicability in complex laminate structures. First, it requires the structure to be dimensionally reduced to a surface, which could be a complex task in itself [22]. Even if the structure is successfully reduced, modeling an assembly of multiple plates and shells can be problematic [6]. In addition, due to dimensional reduction, 2D FEA can sometimes completely miss a 3D phenomenon. For example, in the lap joint problem shown in Figure 3, 2D FEA misses the moments due to eccentric forces when the lap joint is reduced to a surface. Finally, different theories for thin and thick plates and shells further complicate 2D FEA [23].

\subsection{Three-dimensional FEA}

In theory, three-dimensional FEA using layered elements will accurately simulate deformation in laminate structures. In practice, however, using solid elements is expensive, and they also might give inaccurate results due to locking. As a compromise, several hybrid methods that incorporate two-dimensional plate and shell behaviors in 3D FEA have been proposed. For example, Solid-shell elements are 3D elements that use Assumed Natural Strain method to deform like plates and shells $[6,24]$. Their three dimensional nature is well suited for interfacing with other solid elements in assemblies. These elements, however, still require mid-surface extraction and also cannot simulate behaviors other than plate and shell behaviors.

Continuum solid-shell elements, unlike solid-shell elements, are standard displacement based elements, but use advanced finite element techniques like Assumed Strain Method and Enhanced Strain Method to improve their performance for thin structures [10, 11]. The higher the number of assumed and enhanced parameters, the better is the element's performance, but at the expense of generality [10, 25]. Although these elements do not require mid-surfaces explicitly, they must conform to the geometry of the laminate, with their $z$ direction aligned to the transversal direction of structure's offset thickness, because the in-plane and out-of-plane behaviors are assumed or enhanced differently. We refer to such elements as conforming layered elements (Figure 1b). These elements, just like layered elements, are still expensive for FEA of laminates. 
Aligning element's $z$ direction to the laminate's transverse thickness direction also simplifies volume integration. If not aligned, plies can intersect elements at arbitrary angles and require computation of intersections between individual plies and elements, which is both non-trivial and computationally expensive. For this reason, 3D FEA of laminates using non-conforming mesh becomes less attractive, despite its advantages over using conforming mesh [26]. For completeness, we mention that several other methods have been proposed $[5,16,17,27]$, but they either lack generality, or suffer from one or more of the challenges mentioned above.

\section{Material Models for Composite Laminates}

In this section, we briefly summarize the classical theories used to establish the constitutive relationships in a laminate, and derive $A B D$-equivalent material models which remain valid under identical assumptions.

\subsection{Constitutive Relations for Orthotropic Plies}

Plies made of uni-directional fibers are generally modelled as transversely isotropic materials, but we are considering a more general orthotropic model for the plies[4]. To characterize a material in linear elasticity, stiffness matrix $\mathbf{C}$ is used, which requires 9 independent elastic constants in case of orthotropic materials. The constitutive relation between stress and strain takes a general form given by[4]

$$
\sigma_{i}=C_{i j} \cdot \epsilon_{j} \quad i, j=1,2, . ., 6,
$$

where, as usual, $\sigma$ is stress and $\epsilon$ is strain. The equation is in contracted notation where $i, j=1,2$, and 3 are $x, y$, and $z$ coordinate axes, while $i, j=4,5$, and 6 are $y z, z x$, andxy planes respectively. Unlike isotropic materials, $\mathbf{C}$ is direction dependent for orthotropic materials and needs to be transformed from its principal material directions to element's coordinate directions. Full matrix form of Equation 2, including the transformation of $\mathbf{C}$ from its principal to a general coordinate system, is given in Appendix A.1.

The plane-stress constitutive relationship for dimensionally reduced laminates is characterized by a $3 x 3$ stiffness matrix $\mathbf{Q}[4]$ and is given as:

$$
\sigma_{i}=Q_{i j} \cdot \epsilon_{j} \quad i, j=1,2,3,
$$

where $i, j=1$ and2 stand for $x a n d y$ axes respectively, while 3 stand for the $x y$ plane. Full matrix form of the equation is in Appendix A.2.

In thick plates, out-of-plane shear strains $\epsilon_{y z}$ and $\epsilon_{x z}$ are significant, and therefore, outof-plane shear stiffness, in addition to in-plane stiffness, are needed to characterize a ply. In an arbitrary coordinate system, shear stresses $\sigma_{y z}$ and $\sigma_{x z}$ are related to shear strains $\epsilon_{y z}$ and $\epsilon_{x z}$ as [5]:

$$
\left[\begin{array}{l}
\sigma_{4} \\
\sigma_{5}
\end{array}\right]=\left[\begin{array}{ll}
Q_{44} & Q_{45} \\
Q_{45} & Q_{55}
\end{array}\right] \cdot\left[\begin{array}{l}
\epsilon_{4} \\
\epsilon_{5}
\end{array}\right]
$$

where indices 4 and 5 stand for planes $y z$ and $x z$ respectively. 
Let us now assume that, in a laminate, $z$ direction is the thickness direction for both the laminate and its plies, which is also the third principal direction of the orthotropic ply materials. Recall that plate theory assumes that the thickness of a plate in stretching and pure bending remains constant, or in other words, Poisson's ratios $\nu_{x z}$ and $\nu_{y z}$ are zero[6]. This assumption reduces the general stress-strain relation in Equation 2 to

$$
\left[\begin{array}{c}
\sigma_{x} \\
\sigma_{y} \\
\tau_{x y} \\
\tau_{y z} \\
\tau_{x z} \\
\sigma_{z}
\end{array}\right]=\left[\begin{array}{cccccc}
Q_{11} & Q_{12} & Q_{13} & 0 & 0 & 0 \\
Q_{12} & Q_{22} & Q_{13} & 0 & 0 & 0 \\
Q_{13} & Q_{23} & Q_{33} & 0 & 0 & 0 \\
0 & 0 & 0 & Q_{44} & Q_{45} & 0 \\
0 & 0 & 0 & Q_{45} & Q_{55} & 0 \\
0 & 0 & 0 & 0 & 0 & E_{3}
\end{array}\right] \cdot\left[\begin{array}{c}
\epsilon_{x} \\
\epsilon_{y} \\
\gamma_{x y} \\
\gamma_{y z} \\
\gamma_{x z} \\
\epsilon_{z}
\end{array}\right],
$$

where the indices $C_{i j}$ are reordered to match those of $Q_{i j}$. Also, note that $C_{33}$ reduces to $E_{3}$, the Young's Modulus in the third principal material direction.

Setting transverse Poisson's ratios to zero is invalid when 3D stresses, including interlaminar stresses, in laminate structures are being computed. However, the assumption is valid for ABD-equivalent material models because they are based on plate/shell assumptions for laminates which ignore interlaminar effects. As mentioned in Section 1.1, plate/shell assumptions are reasonable assumptions for regions away from boundaries and discontinuities in laminates, where interlaminar stresses are not significant.

\subsection{Constitutive Model for Laminates, or Lamination Theories}

The classical lamination plate theory (CLPT) assumes that laminates can only undergo stretching and pure bending. Therefore, strain $\epsilon_{i}$ at any point in the laminate can be related linearly to strain $\epsilon_{i}^{o}$ and curvature $\kappa_{i}$ at the mid-plane and is given as [4]:

$$
\epsilon_{i}=\epsilon_{i}^{o}+z \cdot \kappa_{i} \quad \mathrm{i}=1,2,3,
$$

where $z$ is the distance of the point from the mid-plane. Figure $2 \mathrm{C}$ shows the plot of $\epsilon_{1}$ at a typical cross section of a laminate.

Since the amounts of stretching and bending in a plate depend only on the net forces and moments, the stresses in the laminate's cross-section can be reduced to just mid-plane forces $N_{i}$ and moments $M_{i}$. This is done by integrating stresses $\sigma_{i}$ over the thickness of the laminate from $\frac{-t}{2}$ to $\frac{t}{2}$ and is given as:

$$
N_{i}=\int_{-\frac{t}{2}}^{\frac{t}{2}} \sigma_{i} d z \quad \text { and } \quad M_{i}=\int_{-\frac{t}{2}}^{\frac{t}{2}} \sigma_{i} z d z .
$$

Combining this equation with plane-stress constitutive relationship from Equation 3 and strain field from Equation 6 leads to the so-called ABD matrix model for laminates:

$$
\left[\begin{array}{r}
\mathbf{N} \\
\mathbf{M}
\end{array}\right]=\left[\begin{array}{ll}
\mathbf{A} & \mathbf{B} \\
\mathbf{B} & \mathbf{D}
\end{array}\right] \cdot\left[\begin{array}{c}
\epsilon^{o} \\
\kappa
\end{array}\right]
$$




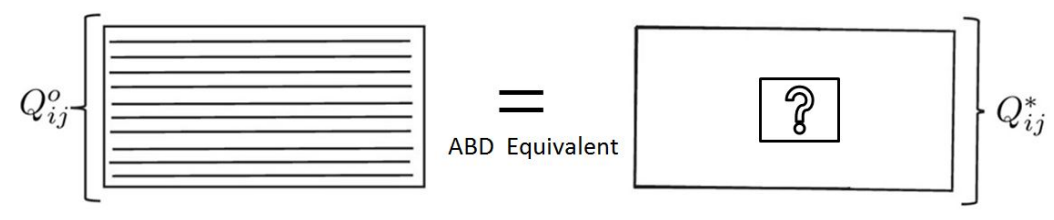

Figure 4: Original laminate with material properties $Q_{i j}^{o}$ can be replaced by a new material model $Q_{i j}^{*}$ that is $A B D$ equivalent to $Q_{i j}^{o}$.

The individual coefficients of $\mathbf{A}, \mathbf{B}$ and $\mathbf{D}$ matrices for indices $i=1,2$, and 3 are given as:

$$
A_{i j}=\int_{-\frac{t}{2}}^{\frac{t}{2}} Q_{i j} d z, \quad B_{i j}=\int_{-\frac{t}{2}}^{\frac{t}{2}} Q_{i j} z d z, \quad D_{i j}=\int_{-\frac{t}{2}}^{\frac{t}{2}} Q_{i j} z^{2} d z .
$$

The details of the derivation can be found in Appendix Appendix B. Intuitively, A and $\mathbf{D}$ are extensional and bending components of the stiffness respectively, while $\mathbf{B}$ couples stiffness between bending and stretching that occurs in a laminate if its material properties are asymmetrical about its mid-plane. If $\mathbf{B}$ is a non-zero matrix, a normal pull in $x$ or $y$ direction can lead to bending and vice versa.

The out-of-plane shear stresses can also be reduced to mid-plane shear forces $\Gamma_{4}$ and $\Gamma_{5}$ using a procedure very similar to the one used to reduce the in-plane stresses to mid-plane forces $N_{i}$ in Equations 7 and 8, leading to

$$
\left[\begin{array}{l}
\Gamma_{4} \\
\Gamma_{5}
\end{array}\right]=K \cdot\left[\begin{array}{ll}
A_{44} & A_{45} \\
A_{45} & A_{55}
\end{array}\right] \cdot\left[\begin{array}{l}
\epsilon_{4} \\
\epsilon_{5}
\end{array}\right]
$$

Here, strains $\epsilon_{4}$ and $\epsilon_{5}$ are assumed constant in the $z$ direction, and any deviation from the actual field is corrected using a correction factor $K$ [5]. The extensional shear stiffness coefficients $A_{44}, A_{45}$, and $A_{55}$ are defined as $A_{i j}$ in Equation 9.

\subsection{ABD-Equivalent Material Models of Laminate}

If we accept that ABD matrices are an accurate approximation of a laminate's behavior, it stands to reason that any two material models that result in identical ABD matrices should be deemed equivalent. In fact, multiple material models with identical ABD matrices exist, forming an equivalence class of material models. From this class, we can seek some of the simplest models, which can be used to replace the original laminate in the 3D integration procedure in FEA (Figure 4).

Any new $A B D$-equivalent model must satisfy the following equivalence relationship:

$$
\begin{aligned}
A_{i j}^{o}=\int_{-\frac{t}{2}}^{\frac{t}{2}} Q_{i j}^{o} \quad d z & =\int_{-\frac{t}{2}}^{\frac{t}{2}} Q_{i j}^{*} d z, \\
B_{i j}^{o}=\int_{-\frac{t}{2}}^{\frac{t}{2}} Q_{i j}^{o} z d z & =\int_{-\frac{t}{2}}^{\frac{t}{2}} Q_{i j}^{*} z d z, \\
D_{i j}^{o}=\int_{-\frac{t}{2}}^{\frac{t}{2}} Q_{i j}^{o} z^{2} d z & =\int_{-\frac{t}{2}}^{\frac{t}{2}} Q_{i j}^{*} z^{2} d z,
\end{aligned}
$$




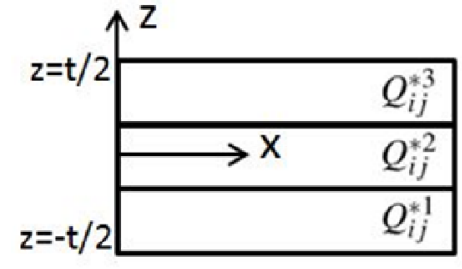

(A)

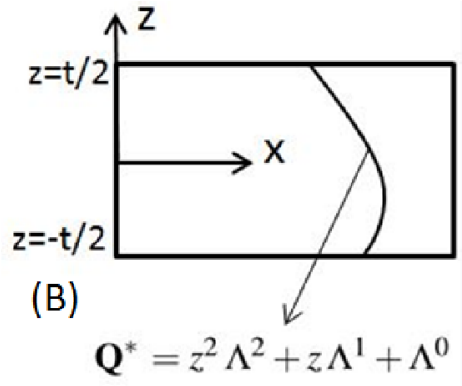

Figure 5: A) A 3-ply ABD-equivalent laminate model with stiffness matrix $Q_{i j}^{* k}$ for the $k^{\text {th }}$ ply. B) A graded ABD-equivalent material with its properties varying quadratically

where the original laminate $Q_{i j}^{o}$ defines the matrices $A_{i j}^{o}, B_{i j}^{o}$ and $D_{i j}^{o}$, and the new material model $Q_{i j}^{*}$ results in the same $\mathrm{ABD}$ matrices.

Since the above integral equations are a system of three equations for each entry of ABD matrices, they can be completely determined by a material model $Q_{i j}^{*}$ that varies in the thickness direction, and the variation is fully specified by 3 or more independent coefficients. There are infinitely many such models, and any two of them are interchangeable if the assumptions made in lamination theory hold. In other words, for the purpose of finite element analysis, any arbitrarily complex laminate with numerous plies can be replaced by a much simpler material model yielding identical results. Furthermore, since the new model is virtual, it is not subject to manufacturing constraints and doesn't necessarily have to be ply-based.

For demonstration, we choose two such material models: a 3-ply laminate and a quadratically graded material model. In the next two subsections, the in-plane material properties of the two ABD-equivalent models respectively are derived, followed in Section 3.3.3 by the derivation of out-of-plane material properties that are common for both the models.

\subsubsection{Three-ply Material Model}

In the equivalence class of material models with given ABD matrices, a 3-ply material model is the simplest ply-based model. Figure 5A shows a 3-ply model with $Q_{i j}^{* k}$ as the material properties of the $k^{\text {th }}$ ply, and for simplicity, each ply is assumed to be of equal thickness. With these assumptions, Equation 11 can be solved for material properties $Q_{i j}^{* k}$, and in terms of $A B D$ matrices of the given laminate, are given as:

$$
Q_{i j}^{* 1}=\frac{36 D_{i j}^{o}-18 t B_{i j}^{o}-t^{2} A_{i j}^{o}}{8 t^{3}}, Q_{i j}^{* 2}=\frac{13 t^{2} A_{i j}^{o}-36 D_{i j}^{o}}{4 t^{3}}, Q_{i j}^{* 3}=\frac{36 D_{i j}^{o}+18 t B_{i j}^{o}-t^{2} A_{i j}^{o}}{8 t^{3}}
$$

where $t$ is the laminate's total thickness and indices $i, j=1,2$, and3. Clearly, there always exists a unique 3-ply laminate that is ABD-equivalent to the original laminate. Note that only the top and bottom plies depend on the $\mathbf{B}$ matrix and capture any material asymmetry about the mid-plane. If the original laminate is symmetrical, the $\mathbf{B}$ matrix is zero, and the two plies $Q_{i j}^{* 1}$ and $Q_{i j}^{* 3}$ are identical. 


\subsubsection{Quadratically Graded Anisotropic Material}

Instead of a ply-based model, we can also replace the original laminate by a continuously varying, or graded, material. Since there are 3 equations to be satisfied, a quadratic variation with 3 independent coefficients $\Lambda_{i j}^{k}$ with $k=1,2,3$ is sufficient. A quadratically varying material model is shown in Figure $5 \mathrm{~B}$ and is given as:

$$
Q_{i j}^{*}=z^{2} \Lambda_{i j}^{2}+z \Lambda_{i j}^{1}+\Lambda_{i j}^{0} .
$$

$\Lambda_{i j}^{k}$ can be found from the equivalence relations in Equation 11, and are given as

$$
\Lambda_{i j}^{0}=\frac{15\left(12 D_{i j}^{o}-t A_{i j}^{o}\right)}{t^{5}}, \quad \Lambda_{i j}^{1}=\frac{12 B_{i j}^{o}}{t^{3}}, \quad \Lambda_{i j}^{2}=\frac{3\left(3 t A_{i j}^{o}-20 D_{i j}^{o}\right)}{4 t^{3}} .
$$

Interestingly, coefficients $\Lambda_{i j}^{k}$ take different roles in the material model: together, the quadratic coefficient $\Lambda_{i j}^{2}$ and the constant coefficient $\Lambda_{i j}^{0}$ capture the bending and in-plane stiffness, while the linear coefficient $\Lambda_{i j}^{1}$ captures the coupling stiffness of the given laminate. Again, there is a unique quadratically varying graded material for the given $A B D$ matrices.

\subsubsection{Transverse Material Properties of the ABD-Equivalent Material Models}

In addition to in-plane, we also require out-of-plane material properties to completely characterize the ABD-equivalent models. These out-of-plane, or transverse, material properties can be derived using approaches similar to above, and are common for both the ABD-equivalent models.

From Equations 9 and 10, the transverse shear properties of a laminate are given by the extensional stiffness matrix $A_{i j}$ for $i, j=4 a n d 5$. Since we are only interested in the equivalent material behavior, transverse shear stiffness $Q_{i j}^{* k}$ can be assumed constant along the laminate's thickness. When substituted in Equation 11, $Q_{i j}^{*}$ are obtained as the average values of $A_{i j}^{o}$ over the laminate's thickness $t$ :

$$
Q_{i j}^{*}=\frac{A_{i j}^{o}}{t} \quad \text { for } i, j=4,5 \text {. }
$$

Transverse normal stiffness of laminates, which is not at all required for 2D FEA, is needed for the ABD-equivalent models. From Equation 5, transverse normal stiffness for plate structures reduces to Young's Modulus $E_{3}$. It is well known that for thin layered structures, the resultant out-of-plane Young's Modulus $E_{3}^{o}$ can be approximated as the harmonic average of the Young's Modulus $E_{3}^{o k}$ of the individual plies of the original laminate[28, 4]. Again, since we are only interested in the equivalent material behavior of the proposed ABD-equivalent models, their Young's Modulus $E_{3}^{*}$ can be assumed constant throughout the laminate thickness. This assumption makes $E_{3}^{*}$ identical to $E_{3}^{o}$ :

$$
\frac{1}{E_{3}^{*}}=\frac{1}{E_{3}^{o}}=\sum_{k=1}^{k=n} \frac{h^{k}}{E_{3}^{o k}},
$$

where $h^{k}$ is the thickness of the $k^{t h}$ ply and $n$ is the total number of plies in the original laminate. 


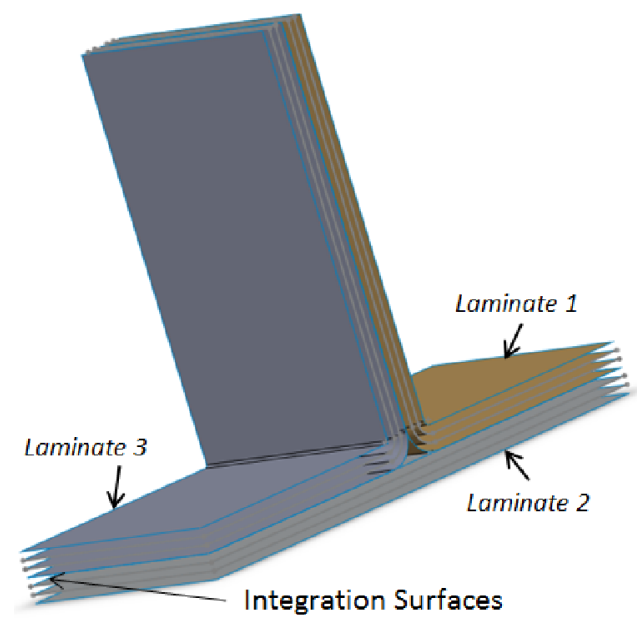

Figure 6: Laminate structure in Figure 1 replaced by integration surfaces.

To summarize, we can efficiently construct various virtual material models that are $A B D$ equivalent to the original laminate. If the usual lamination theory assumptions hold for the original laminate, these virtual models will result in identical stiffness matrices during any finite element analysis procedure. Any one of these models can be used during analysis; however, some models could be easier to implement than others in a particular system. For example, the 3-ply laminate model is straightforward to implement in systems which already support representation of laminates. On the other hand, the graded material model can be used to analyze laminates in systems that are meant for graded materials, but do not support laminates. In the next section, we discuss implementation of these two ABDequivalent material models and demonstrate their validity.

\section{Implementation}

Laminates, as proposed by the current and emerging standards, are commonly represented as a base surface and an associated layup table with an entry for each ply [29]. Base surfaces are generally the tooling surfaces on which plies are laid, and the table specifies the order, materials, and fiber directions of the individual plies. In systems supporting laminates based on the above standard, implementing the 3-ply model is straightforward: the original layup table with any number of entries is replaced by a new table with only 3 entries. However, for implementing the graded material model, instead of a table for plies, coefficients $\Lambda_{i j}$ defining the quadratically graded material in Equation 13 are required.

We implemented the ABD-equivalent models in a meshfree system called Scan and Solve (SnS) [26]. In SnS, displacements and stresses are approximated using multi-variate Bspline functions that are constructed over a uniform Cartesian grid. This choice of the basis function addresses the concerns of shear locking as well as numerical ill-conditioning of stiffness matrix for a wide range of laminate thicknesses. More details about Scan and Solve can be found in the reference [26]. 


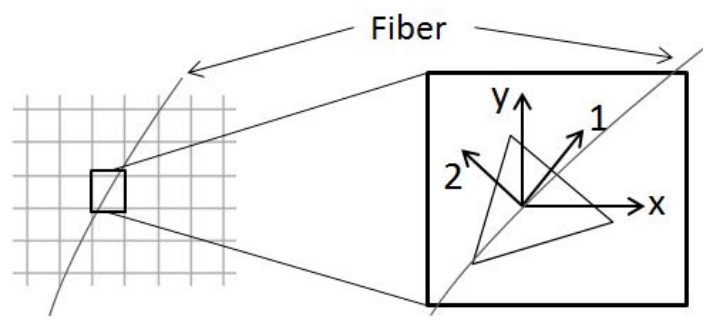

(A)

(B)

Figure 7: A) An xy cross-section of the Cartesian grid, and an arbitrary fiber in that cross-section. B) Figure zooms in one of the grid elements and shows a triangle that is being integrated. From the fiber orientation, the material principal directions 1 and 2 are found, which are not aligned to element directions $x$ and $y$ in general.

During finite element analysis, the ABD-equivalent material models come into picture while computing element stiffness matrices given in Equation 1. If the mesh is conforming and the element's $\mathrm{z}$ axis is aligned to the laminate's thickness direction (Figure 1b), computing volume integration for the 3-ply model is straightforward. This is because, in a conforming mesh, an individual ply's exact location can be completely determined by its position in the $\mathrm{z}$ direction. However volume integration is more involved for a non-conforming mesh. Plies can intersect a grid element at arbitrary angles (Figure 1c and Figure 1d), and also in structures made of multiple laminates, more than one laminate can intersect an element. As a result, computing intersection of each ply with an element can be both complicated as well as expensive. Therefore, for an ease of implementation, we approximate volume integration by integration over surfaces: each laminate is replaced by a set of surfaces parallel to the tooling surface (Figure 6). These surfaces, which we call integration surfaces, can be easily generated as tooling surface's offsets, a standard geometric operation in a CAD software. The location of these integration surfaces in the laminate's thickness direction can be obtained using one of the quadrature rules, and in the current implementation, we use the Lobatto quadrature rules [30]. In addition to simplifying volume integration, this integration scheme also makes implementation of the ABD-equivalent graded model much easier: since an integration surface is an offset at a constant distance from the laminate's mid-plane, coefficients $\Lambda_{i j}$ of the graded material (Equation 13) are also constant within that integration surface and, therefore, need to be computed once. Integration over each surface is done by first triangulating it, and then integrating the obtained triangles using Gauss quadrature rules. The triangles are constrained to conform to the Cartesian grid, or in other words, each triangle completely lies within an element of the Cartesian grid.

While integrating over the triangles, we also need to transform the material matrix $\mathbf{Q}^{*}$ from its principal coordinate system to the element coordinate system. As explained in Figure 7, for every triangle, we transform $\mathrm{Q}^{*}$ once for the triangle's centroid, and use the transformed properties $\mathbf{Q}^{*^{\prime}}$ for all the quadrature points of that triangle. The transformation relation is explained in detail in Appendix A.1 and in matrix form is given as:

$$
\mathrm{Q}^{*^{\prime}}=\mathrm{G}^{T} \cdot \mathrm{Q}^{*} \cdot \mathrm{G}
$$


where $\mathbf{G}$ is the transformation matrix.

Finally, we assume plate behavior everywhere in our simple benchmark laminate problems, with no distinction between plate and non-plate regions. This assumption is justified because we compare our results with those obtained using dimensionally-reduced models of laminates. We skip rest of the implementation details as they are not directly relevant to the current work.

\section{Numerical Test Results}

In this section, we compare results of linear static analysis computed using ABD-equivalent materials to results in the standard reference text[5], as well as to results computed using commercial software SolidWorks[31] and ANSYS [32]. Since we are using 3D meshfree FEA implementation, we do not expect it to be more efficient than highly optimized commercial 2D FEA solutions. Our only goal is to demonstrate the accuracy of ABD-equivalent material models in comparison with other analytical and computational methods that rely on the ABD formulation. This also explains why we do not compare the accuracy of our results to more accurate models based on higher-order lamination theories or full 3D elasticity formulations.

We are considering four benchmark problems: a rectangular plate, a cylindrical shell, a cylindrical roof, and a lap joint. The first three structures are single multi-ply laminates, while the lap joint is a bonded assembly of two laminates. For each of the test structures, we consider the following three configurations:

1. Cross-ply laminates $[0 / 90]_{n}$,

2. Angle-ply laminates $[-45 / 45]_{n}$,

3. 50-ply laminates (Appendix Appendix D).

Cross-ply and angle-ply laminate configurations are often standard configurations. The 50ply laminate was chosen to test if the proposed method scales for a large number of plies. The plies in the 50-ply laminate were selected randomly, and their orientations are given in Appendix Appendix D. We restricted the number of plies to 50 because that was the maximum number of plies supported by SolidWorks. The three laminate configurations are asymmetrical about their mid-plane, and therefore show stretching-bending coupling, as predicted by the classical lamination theory. All plies in each laminate are assumed to be made of one of the two materials:

Material 1: $E_{1}=25.0 e^{6} p s i, E_{2}=E_{3}=1.0 e^{6} p s i, \nu_{12}=0.25, \nu_{23}=\nu_{13}=0.0, G_{12}=G_{13}=$ $5.0 e^{5}$ psi and $G_{23}=2.0 e^{5}$ psi,

Material 2: $E_{1}=7.5 e^{6} p s i, E_{2}=E_{3}=2.0 e^{6} p s i, \nu_{12}=0.25, \nu_{23}=\nu_{13}=0.0, G_{12}=$ $1.25 e^{6} p s i$, and $G_{13}=G_{23}=0.625 e^{6} p s i$,

where $E, \nu$ and $G$ are Young's Modulus, Poisson's Ratio and Shear Modulus respectively, and indices 1,2 and 3 represent the three principal material directions. These materials are 


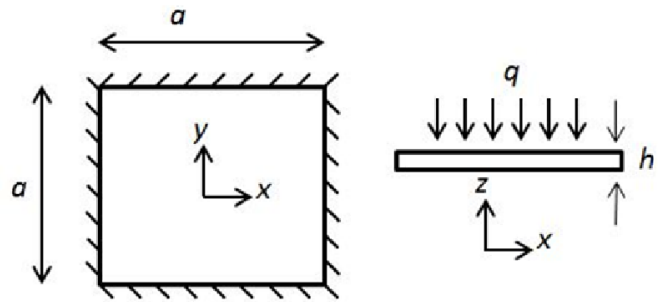

(A)

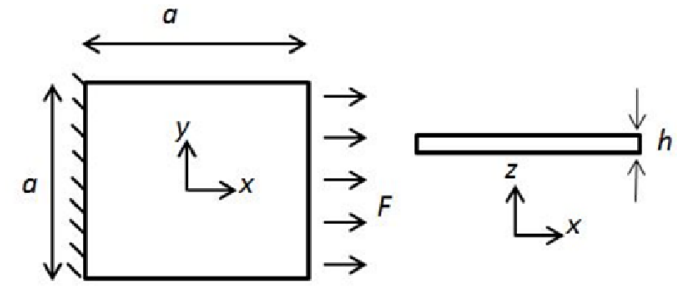

(B)

Figure 8: A) Plate with geometry parameters $a=10 \mathrm{in}$ and $h=1,0.1,0.01 \mathrm{in}$, clamped from all four sides with a surface pressure, $q=100 \mathrm{psi}$. B) Plate with $a=10 \mathrm{in}$ and $h=0.1 \mathrm{in}$, fixed on one end with a force, $F=1.0 e^{4} l b f$ on the opposite end.

identical to those used in [5] that we use for comparison, except we set $\nu_{23}$ and $\nu_{13}$ to be zero.

Finally we note that the elements available for analyzing laminates in the three systems are different:

Solidworks: we used two-dimensional parabolic triangular shell elements.

ANSYS: we used two-dimensional Shell181 elements for analyzing single laminate structures, and Solid186 elements for analyzing the lap joint. Shel181 are 4 node elements with 3 displacement and 3 rotational degrees of freedom at each node. A penalty method is used to relate the independent rotational degrees of freedom about the normal (to the shell surface) with the in-plane components of displacements. Solid186 elements are 20-node layered solid elements that exhibit quadratic displacement behavior [32].

Scan\&Solve: each benchmark problem is solved using 1000 and 3000 second-order trivariate B-spline functions on a uniform Cartesian non-conforming grid. The Lobatto quadrature rule implies that 3 integration surfaces per ply for the 3-ply laminate model, and 4 integration surfaces for the quadratically graded laminate model, are sufficient for full integration.

\subsection{Clamped Rectangular Plate}

Our first benchmark problem is a plate clamped on all four sides, and a normal pressure on the top surface (Figure 8A). A plate under these boundary conditions shows pure bending, with maximum displacement at the center of the plate. This particular problem was chosen to test if second order B-spline basis in Scan and Solve can successfully capture bending in thin structures, since conventional 3D basis functions tend to underestimate bending deformations due to locking. The plate was made of Material 1, and consisted of 10 plies laid in cross-ply and angle-ply configurations.

Table 1 compares the maximum displacements using ANSYS, SolidWorks (SW), and proposed method $(\mathrm{SnS})$ for different laminates. Tests were done for three different aspect ratios: thin $(\mathrm{a} / \mathrm{h}=1000)$, moderately thick $(\mathrm{a} / \mathrm{h}=100)$, and thick $(\mathrm{a} / \mathrm{h}=10)$. For both 
Table 1: Maximum displacement value in inches for plate problem in Figure 8A for different cases.

\begin{tabular}{rllcccccc}
\hline Laminate & Thickness & $a / h$ & ANSYS & SW & \multicolumn{2}{c}{ SnS-3-Ply } & \multicolumn{2}{c}{ SnS-Graded } \\
\hline (Number of elements) & & $10 \mathrm{k}$ & $1 \mathrm{k}$ & $1 \mathrm{k}$ & $3 \mathrm{k}$ & $1 \mathrm{k}$ & $3 \mathrm{k}$ \\
\hline \multirow{2}{*}[0/90]{$_{5}$} & 1 & 10 & $4.748 e^{-3}$ & $4.058 e^{-3}$ & $3.855 e^{-3}$ & $3.762 e^{-3}$ & $4.567 e^{-3}$ & $3.755 e^{-3}$ \\
& 0.1 & 100 & $1.543 e^{+0}$ & $1.550 e^{+0}$ & $1.532 e^{+0}$ & $1.543 e^{+0}$ & $1.532 e^{+0}$ & $1.543 e^{+0}$ \\
& 0.01 & 1000 & $1.510 e^{+3}$ & $1.552 e^{+3}$ & $1.145 e^{+3}$ & $1.661 e^{+3}$ & $1.145 e^{+3}$ & $1.662 e^{+3}$ \\
\hline \multirow{2}{*}[-45/45]{$_{5}$} & 1 & 10 & $5.094 e^{-3}$ & $4.360 e^{-3}$ & $4.057 e^{-3}$ & $4.152 e^{-3}$ & $4.880 e^{-3}$ & $4.050 e^{-3}$ \\
& 0.1 & 100 & $1.629 e^{+0}$ & $1.620 e^{+0}$ & $1.597 e^{+0}$ & $1.611 e^{+0}$ & $1.598 e^{+0}$ & $1.611 e^{+0}$ \\
& 0.01 & 1000 & $1.581 e^{+3}$ & $1.578 e^{+3}$ & $1.163 e^{+3}$ & $1.684 e^{+3}$ & $1.163 e^{+3}$ & $1.685 e^{+3}$ \\
\hline
\end{tabular}

Table 2: Von Mises stress at the mid-point $(\mathrm{x}=0, \mathrm{y}=0)$ of the top face of the plate in Figure 8A

\begin{tabular}{rllcccccc}
\hline Laminate & Thickness & $a / h$ & ANSYS & \multicolumn{2}{c}{ SW } & \multicolumn{2}{c}{ SnS-3-Ply } & \multicolumn{2}{c}{ SnS- Graded } \\
\hline (Number of elements) & & $10 \mathrm{k}$ & $1 \mathrm{k}$ & $1 \mathrm{k}$ & $3 \mathrm{k}$ & $1 \mathrm{k}$ & $3 \mathrm{k}$ \\
\hline \multirow{2}{*}[0/90]{$_{5}$} & 1 & 10 & $2.42 e^{3}$ & $2.43 e^{3}$ & $2.50 e^{3}$ & $2.40 e^{3}$ & $3.20 e^{3}$ & $2.35 e^{3}$ \\
& 0.1 & 100 & $2.54 e^{5}$ & $2.55 e^{5}$ & $2.04 e^{5}$ & $2.06 e^{5}$ & $2.05 e^{5}$ & $2.06 e^{5}$ \\
& 0.01 & 1000 & $2.50 e^{7}$ & $2.60 e^{7}$ & $1.60 e^{7}$ & $1.94 e^{7}$ & $1.60 e^{7}$ & $1.93 e^{7}$ \\
\hline \multirow{2}{*}[-45/45]{$_{5}$} & 0.1 & 10 & $2.70 e^{3}$ & $2.66 e^{3}$ & $2.67 e^{3}$ & $2.60 e^{3}$ & $3.20 e^{3}$ & $2.60 e^{3}$ \\
& 0.01 & 100 & $2.25 e^{5}$ & $2.26 e^{5}$ & $1.80 e^{5}$ & $1.82 e^{5}$ & $1.80 e^{5}$ & $1.82 e^{5}$ \\
& & 1000 & $2.25 e^{7}$ & $2.22 e^{7}$ & $1.52 e^{7}$ & $1.70 e^{7}$ & $1.52 e^{7}$ & $1.69 e^{7}$ \\
\hline
\end{tabular}




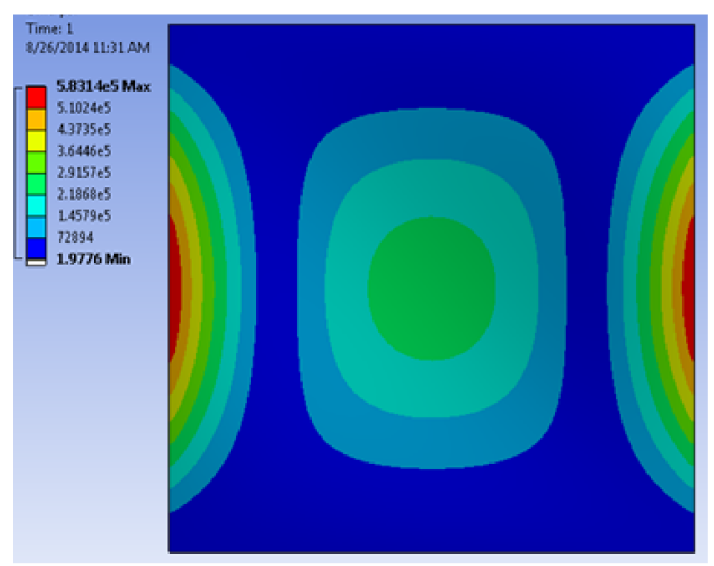

(A1)

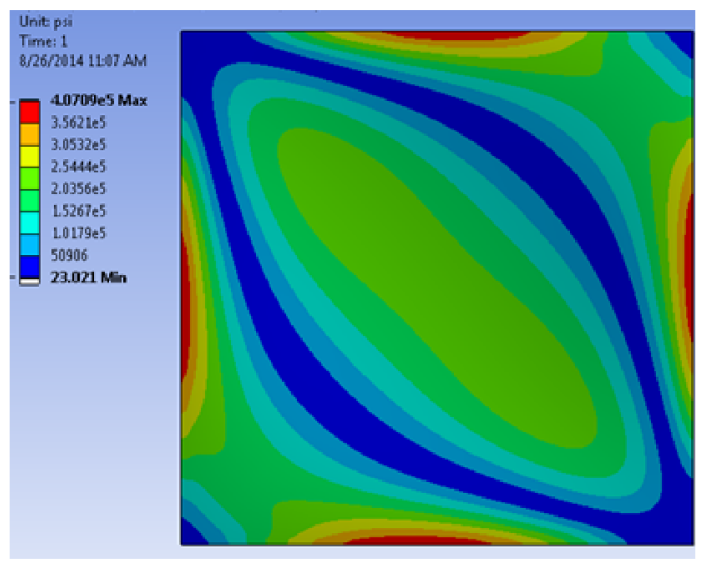

(B1)

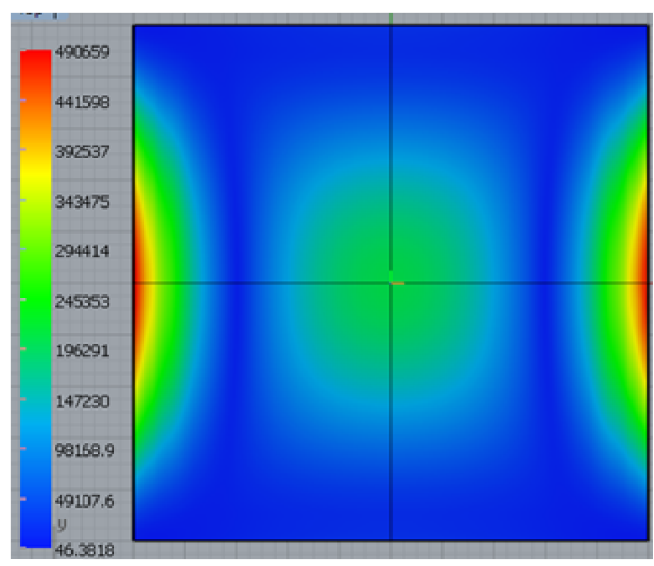

(A2)

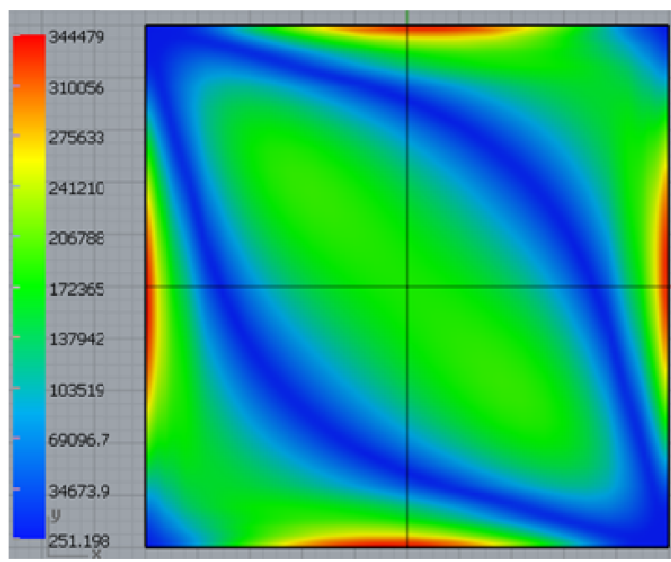

(B2)

Figure 9: Figure compares Von Mises stress for top face of the plate in Figure 8 with aspect ratio 100 for A1) $[0,90]_{5}$ analyzed in ANSYS (Max stress- 5.83e $e^{5}$ 2.[0,90 $]_{5}$ analyzed using the proposed method (Max stress$\left.4.91 e^{5}\right)$. B) $[-45,45]_{5}$ analyzed in ANSYS (Max stress- 4.08e $e^{5}$ 2. $[-45,45]_{5}$ analyzed using the proposed method (Max stress- $3.44 e^{5}$.)

cross-ply and angle-ply laminates with moderate thickness, SnS accurately predicts the maximum displacement values, and the results from all the systems are in close agreement. There are more noticeable differences in the displacements computed by the three systems for thin and thick laminates, e.g., SnS and SW tend to differ by 5\%. Importantly, SnS is not under-predicting displacements for thin plates, suggesting that locking is is not an issue.

We also compare, in Table 2, the Von Mises stresses at the mid-point $(x=0, y=0)$ of the top face for different plates. For both cross-ply and angle-ply laminates, stresses from different methods are in agreement for the thick plate with aspect ratio 10, but there is some deviation for other aspect ratios, which increases with plate aspect ratio. A comparison of the entire stress field for the moderate thickness plate (aspect ratio 100) is shown in Figure 9 for cross-ply and angle ply laminates. The stress patterns match for the two laminates, and the highest stress due to stress concentration are also within $15 \%$ of each other. Solid 

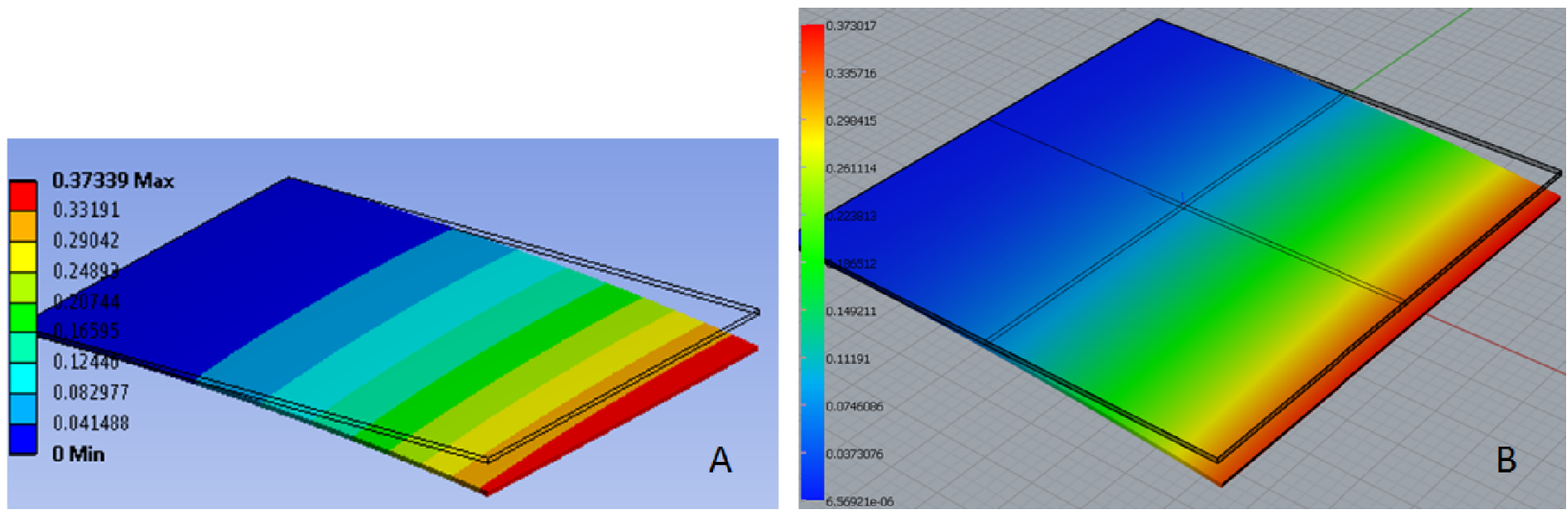

Figure 10: Figure shows deformed plate and color map of out-of-plane displacement obtained using A) ANSYS B) Scan\&Solve with ABD-equivalent 3-ply material model, for a laminate plate made of 50 plies. As expected, the in-plane load leads to out-of-plane bending.

elements generally better capture the stresses near edges as they explicitly model the finite thickness of plates.

Next, we test the same rectangular plate, but made of 50 random plies. The boundary conditions are different from the previous tests; the plate is under in-plane load of $1.0 e^{4}$ lbf on one end and clamped at the opposite end (Figure 8B). This particular problem was chosen to validate two important claims: a) proposed method can handle large number of plies, b) proposed ABD-equivalent material models successfully capture coupling behavior in laminates that are asymmetrical about the mid-plane. Due to this coupling, the in-plane load $F$ will lead to bending and produces out-of-plane deformation. The proposed method did capture this coupling phenomena accurately as shown in Figure 10, which compares the $z$ displacement fields from SnS and and ANSYS. The maximum $z$ displacement are in agreement with 0.3730in in ANSYS and 0.3734in in SnS.

We also carried out a time analysis in order to estimate the net efficiency achieved using proposed method when using 3D FEA. Complete analysis of the 50-ply laminate plate using the ABD-equivalent 3-ply model took 14.9 seconds, out of which 12.8 seconds were spent integrating 9 integration surfaces (3 surfaces per ply). Therefore, an average of 1.42 seconds were spent integrating each surface. This implies that integrating over 150 surfaces in the original 50-ply model would require roughly 215 seconds for the same analysis. The gain in efficiency is even higher when using graded material model, as it needs only 4 integration surfaces in comparison to 9 for the 3-ply laminate. The total time taken for analysis was only 6.8 seconds, decreasing the total computation cost of analysis by more than 30 times. Similar speedup should be expected in any 3D FEA of laminated structures relying on layered elements.

\subsection{Clamped Cylinder With Internal Pressure}

Next, we consider a cylinder, made of Material 2, that is clamped at the two ends and has an internal pressure of $P_{o}$. Details of geometry and boundary conditions are shown in Figure 11. 

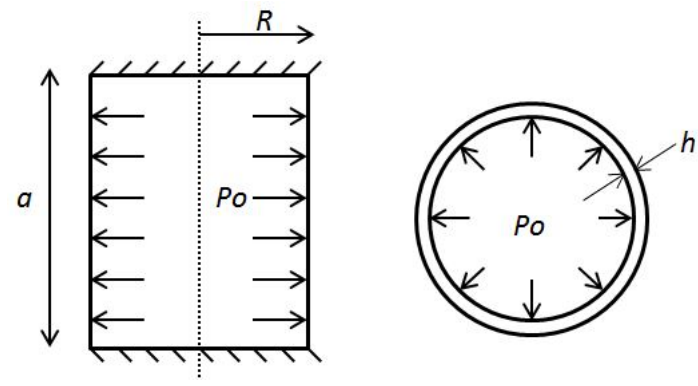

Figure 11: Clamped cylinder with $P_{o}=2.04 k s i, R=20 \mathrm{in}, a=20 \mathrm{in}, h=1 \mathrm{in}$

Table 3: Maximum radial deflection ( $e^{-1}$ in.) for cylindrical shell in Figure 11. Q4 and Q9 results from [5]. SW- SolidWorks, SnS- Proposed Method

\begin{tabular}{rcccccccc}
\hline Laminate & Q4 & Q9 & ANSYS & SW & \multicolumn{2}{c}{ SnS-3-Ply } & \multicolumn{2}{c}{ SnS-Graded } \\
\hline & 16 & 4 & $15 \mathrm{k}$ & $1.2 \mathrm{k}$ & $1 \mathrm{k}$ & $3 \mathrm{k}$ & $1 \mathrm{k}$ & $3 \mathrm{k}$ \\
\hline$[0 / 90]$ & 1.870 & 1.803 & 1.706 & 1.848 & 1.820 & 1.773 & 1.820 & 1.773 \\
{$[-45 / 45]$} & - & - & 2.204 & 2.350 & 2.356 & 2.291 & 2.355 & 2.290 \\
{$[0 / 90]_{5}$} & - & - & 1.719 & 1.830 & 1.814 & 1.776 & 1.815 & 1.776 \\
{$[-45 / 45]_{5}$} & - & - & 2.210 & 2.340 & 2.334 & 2.282 & 2.334 & 2.281 \\
$50-$ ply & - & - & 2.350 & 2.542 & 2.465 & 2.424 & 2.464 & 2.424 \\
\hline
\end{tabular}

The maximum radial deflections from Reddy [5], ANSYS, SolidWorks, and proposed method are compared in Table 3. Elements used by Reddy [5] are Q4 elemens, a fournode (linear) quadrilateral element, and Q9 elements, a nine-node (quadratic) quadrilateral element. The total number of elements used in different methods are specified in the second row of Table 3. The tests were performed for one aspect ratio, but 5 different laminates. Results from all the methods are clearly in agreement. Figure 12 shows the deformed shape, as well as, the color map of displacement value for the cylinder made of 50 plies. Maximum radial displacement using ANSYS is $0.2350 \mathrm{in}$, and using 3-ply material model in Scan and Solve is $0.2419 \mathrm{in}$, which is less than $3 \%$ difference.

\subsection{Barrel Vault Problem}

In this section, we consider another popular benchmark shell problem known as the Barrel vault problem[5]: a cylindrical roof under its own weight. The structure is made of Material 1, and the detailed boundary conditions are shown in Figure 13.

In reference [5], maximum vertical displacements are given for cross-ply and angle-ply laminates with different aspect ratios. We use these values for comparing values obtained using SW, ANSYS, and proposed method in Table 4. Reddy used Q81 elements, which are eighth order element $(p=8)$ with 405 degrees of freedom. For other methods, elements are same as before. Again, there is a fairly close agreement between the results from Reddy [5], 

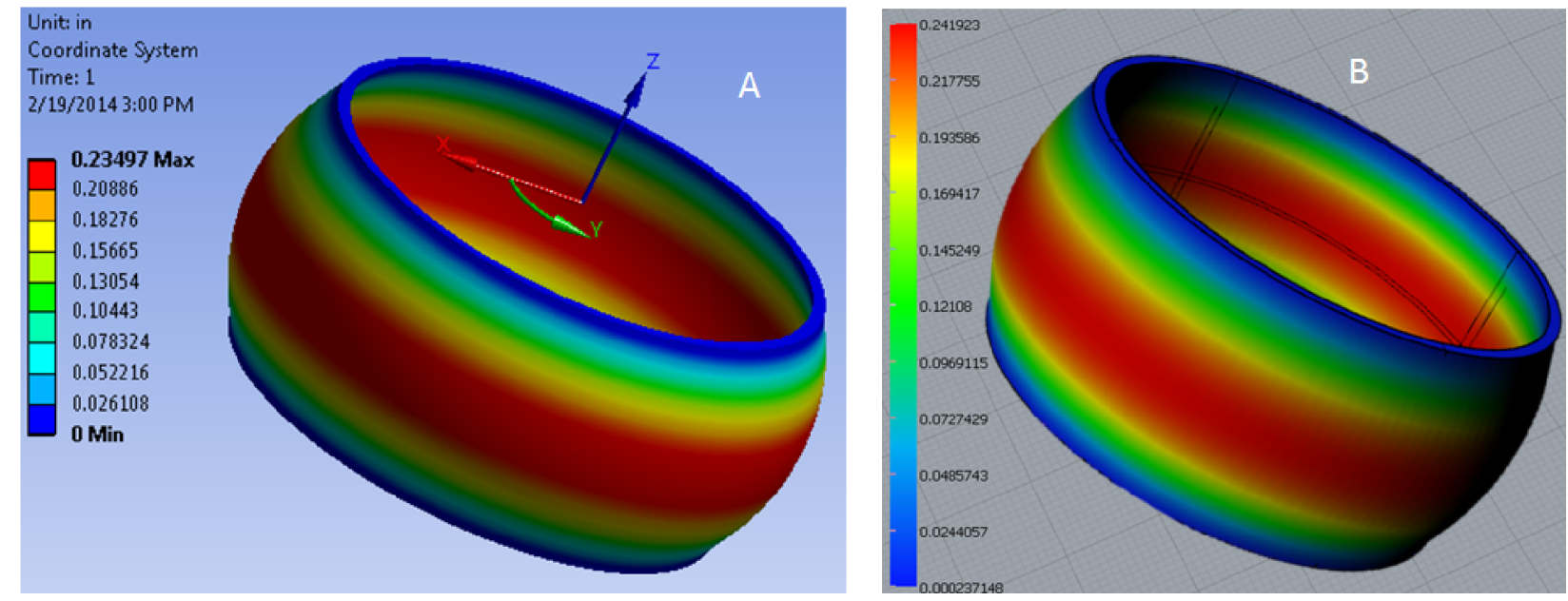

Figure 12: Figure shows deformed cylinder and the color map of radial displacement obtained using A) ANSYS B) Present method, for a laminate cylinder made of 50 plies.

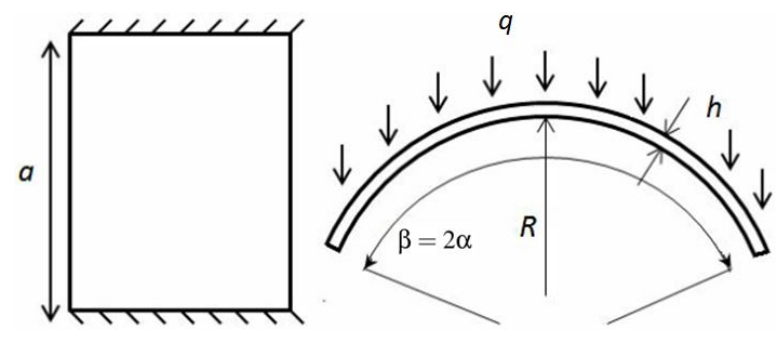

Figure 13: Figure showing barrel vault problem. Vertical pressure is $q=.625 p s i$ and the curved ends are fixed. Other dimension: $\beta=80^{\circ}, R=300 \mathrm{in}, a=600 \mathrm{in}, h=3,6$ and $15 \mathrm{in}$

SolidWorks, ANSYS and proposed method in all cases, including the 50-ply laminate (see Figure 14). The maximum displacement values from Scan\&Solve using 3000 elements and ANSYS are within $2.5 \%$ of each other.

As before, when analysing 50-ply laminates using layered 3D elements, the 3-ply laminate improves efficiency roughly 15 times, whereas the graded material was 30 times more efficient than using the actual laminate layup.

\subsection{Multi-laminate Structure-Lap Joint}

Finally, to show that proposed method can be extended to structures made of multiple laminates, we analyze a lap joint made of two laminates bonded together. Lap joints are very common in real-life structures, and exhibit complex deformation field, even though they have simple geometry. As discussed earlier, direct application of $2 \mathrm{D}$ finite element methods does not capture 3D phenomena in lap joints, and special theories are needed. By contrast, we have shown in our earlier work [21] that 3D FEA using non-conforming mesh can be used to simulate lap joints made of homogeneous materials. We now demonstrate that this approach extends to laminate structures using ABD-equivalent materials.

The geometry and boundary conditions of the lap joint analyzed are shown in Figure 
Table 4: Barrel vault problem- maximum deflection(in.) for cross-ply and angle ply laminates laminates.

\begin{tabular}{|c|c|c|c|c|c|c|c|c|}
\hline Laminate & $\mathrm{R} / \mathrm{h}$ & Reddy & ANSYS & SW & \multicolumn{2}{|c|}{ SnS- 3-Ply } & \multicolumn{2}{|c|}{ SnS- Graded } \\
\hline (No. elem & nts) & 16 & $10 \mathrm{k}$ & $1.2 \mathrm{k}$ & $1 \mathrm{k}$ & $3 \mathrm{k}$ & $1 \mathrm{k}$ & $3 \mathrm{k}$ \\
\hline \multirow{3}{*}[0/90]{} & 100 & $2.339 e^{+0}$ & $2.407 e^{+0}$ & $2.460 e^{+0}$ & $2.307 e^{+0}$ & $2.415 e^{+0}$ & $2.307 e^{+0}$ & $2.416 e^{+0}$ \\
\hline & 50 & $5.082 e^{-1}$ & $5.291 e^{-1}$ & $5.659 e^{-1}$ & $5.480 e^{-1}$ & $5.810 e^{-1}$ & $5.496 e^{-1}$ & $5.503 e^{-1}$ \\
\hline & 20 & $7.292 e^{-2}$ & $7.449 e^{-2}$ & $7.560 e^{-2}$ & $7.877 e^{-2}$ & $8.067 e^{-2}$ & $7.956 e^{-2}$ & $7.016 e^{-2}$ \\
\hline \multirow{3}{*}[-45/45]{} & 100 & $3.597 e^{+0}$ & $3.871 e^{+0}$ & $3.866 e^{+0}$ & $3.411 e^{+0}$ & $3.743 e^{+0}$ & $3.413 e^{+0}$ & $3.699 e^{+0}$ \\
\hline & 50 & $6.760 e^{-1}$ & $7.652 e^{-1}$ & $7.170 e^{-1}$ & $6.675 e^{-1}$ & $7.157 e^{-1}$ & $6.671 e^{-1}$ & $7.780 e^{-1}$ \\
\hline & 20 & $1.205 e^{-1}$ & $1.397 e^{-2}$ & $1.130 e^{-1}$ & $1.061 e^{-1}$ & $1.127 e^{-1}$ & $1.131 e^{-1}$ & $1.386 e^{-1}$ \\
\hline \multirow{3}{*}[0/90]{$_{5}$} & 100 & $1.415 e^{+0}$ & $1.434 e^{+0}$ & $1.564 e^{+0}$ & $1.542 e^{+0}$ & $1.593 e^{+0}$ & $1.540 e^{+0}$ & $1.593 e^{+0}$ \\
\hline & 50 & $2.940 e^{-1}$ & $2.979 e^{-1}$ & $3.270 e^{-1}$ & $3.335 e^{-1}$ & $3.412 e^{-1}$ & $3.337 e^{-1}$ & $3.412 e^{-1}$ \\
\hline & 20 & $5.234 e^{-2}$ & $5.246 e^{-2}$ & $5.370 e^{-2}$ & $5.361 e^{-2}$ & $5.398 e^{-2}$ & $5.361 e^{-2}$ & $5.399 e^{-2}$ \\
\hline \multirow{3}{*}[-45/45]{$_{5}$} & 100 & $1.818 e^{+0}$ & $1.836 e^{+0}$ & $1.955 e^{+0}$ & $1.821 e^{+0}$ & $1.912 e^{+0}$ & $1.821 e^{+0}$ & $1.912 e^{+0}$ \\
\hline & 50 & $4.096 e^{-1}$ & $4.082 e^{-1}$ & $4.089 e^{-1}$ & $3.796 e^{-1}$ & $3.940 e^{-1}$ & $3.799 e^{-1}$ & $3.940 e^{-1}$ \\
\hline & 20 & $1.004 e^{-1}$ & $9.727 e^{-2}$ & $8.959 e^{-2}$ & $7.856 e^{-2}$ & $8.009 e^{-2}$ & $7.857 e^{-2}$ & $8.088 e^{-2}$ \\
\hline 50-ply & 100 & - & $1.4106 e^{0}$ & $1.478 e^{0}$ & $1.391 e^{0}$ & $1.445 e^{0}$ & $1.391 e^{0}$ & $1.445 e^{0}$ \\
\hline
\end{tabular}

Table 5: Maximum displacement value in inches for lap joint in Figure 15. Second row shows the number of elements used.

\begin{tabular}{rccccc}
\hline Laminate & ANSYS & \multicolumn{2}{c}{ SnS- 3-Ply } & \multicolumn{2}{c}{ SnS- Graded } \\
\hline & 760 & $1 \mathrm{k}$ & $3 \mathrm{k}$ & $1 \mathrm{k}$ & $3 \mathrm{k}$ \\
\hline$[0,90]_{5}$ & 1.251 & 1.147 & 1.212 & 1.147 & 1.212 \\
{$[-45,45]_{5}$} & 7.975 & 6.540 & 7.641 & 6.540 & 7.641 \\
50 Plies & 3.304 & 2.937 & 3.194 & 2.938 & 3.195 \\
\hline
\end{tabular}




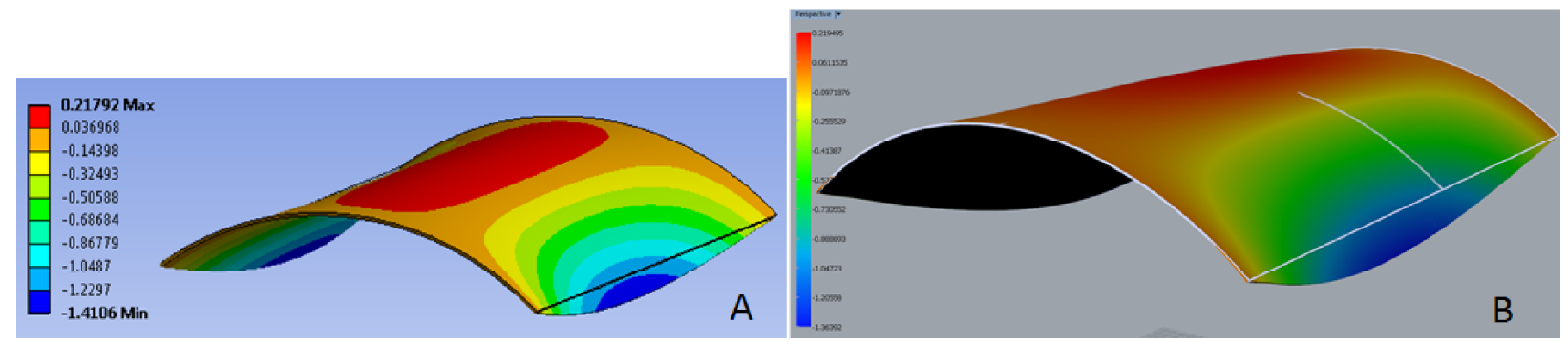

Figure 14: Figure shows deformed shell and color map of out-of-plane displacement obtained using A) ANSYS B) Present method, for a laminate plate made of 50 plies.

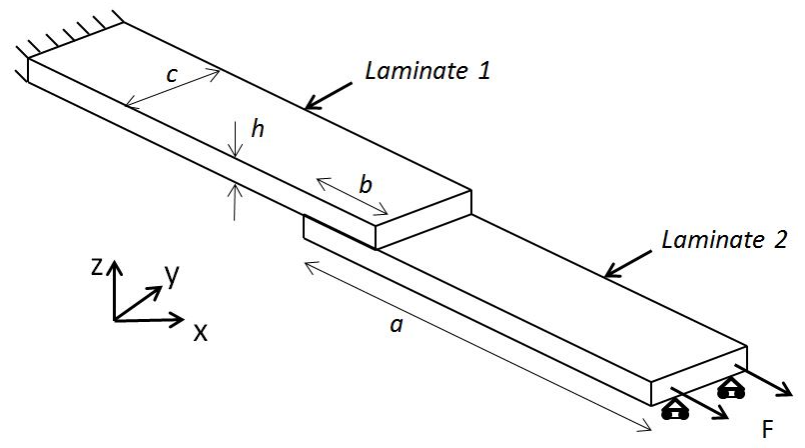

Figure 15: Figure shows a lap joint made of two laminates identical in geometry, with $a=10 \mathrm{in}, b=2 \mathrm{in}$, $c=2.5 \mathrm{in}$ and $h=0.1 \mathrm{in}$. The left end is fully fixed, and the right end is allowed to slide in the $\mathrm{x}$ direction. A force $F=10 e^{4} l b$ is also applied on the right end.

15. The two laminates are made of Material 1, and we have ignored the adhesive layer for simplicity. We compare the results computed in Scan\&Solve using ABD-equivalent materials to those computed in ANSYS using a 20-node layered solid element (since 2D shell elements are not appropriate). Table 4 shows that the maximum displacement values are in close agreement. Figure 16 shows displacement color map as well as the deformed lap joint for the 50-ply laminate. We also compare Von Mises stresses for the lap joint in Figure 17 to confirm if proposed method accurately captures stress concentration near the joint as well as the overall stress field. The maximum stress value at the joint is $3.87 e^{5} p s i$ from ANSYS and $3.32 e^{5}$ psi using the the proposed method which are in agreement considering that the joint region is a region of stress concentration.

For our final test, we show that proposed method also accurately captures coupling phenomena in multi-laminate structure. We analyze the same lap joint, but it is now made of laminates with substantial stretching-bending coupling properties. In laminate [0/90], there is a strong coupling between in-plane stretching and out-of-plane cylindrical bending, while in laminate [-45/45], there is a strong coupling between in-plane stretching and outof-plane twisting. For both the laminates, we compare deformation in the lap joint in two cases: (1) for laminate [0/90], when $0^{\circ}$ plies are bonded together (Figure 18A) and when $90^{\circ}$ plies are bonded together (Figure 18B); (2) for laminate [-45/45], when $45^{\circ}$ plies are bonded together (Figure 19A) and when $-45^{\circ}$ plies are bonded together (Figure 19B). As 


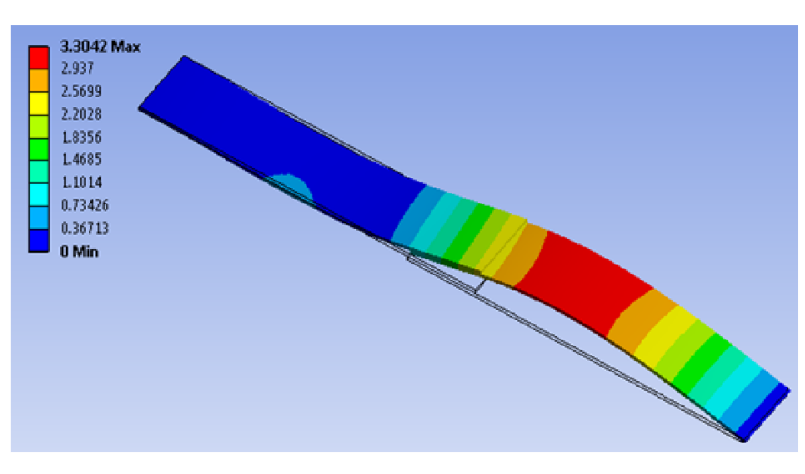

(A)

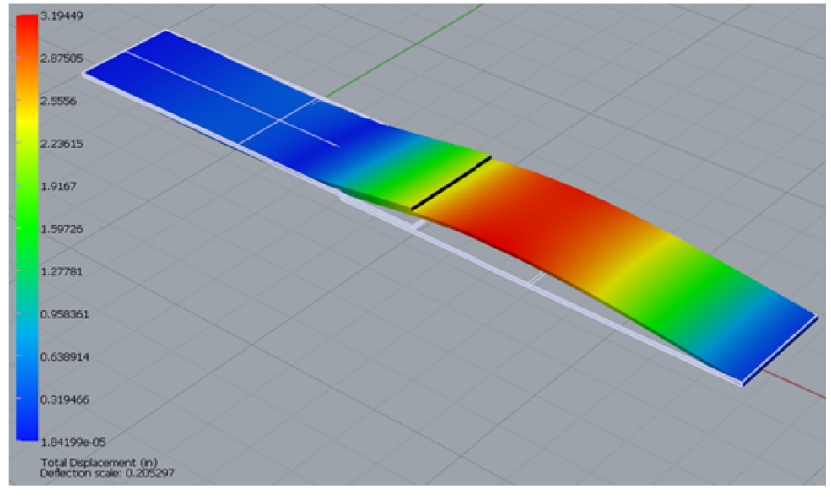

(B)

Figure 16: Figure shows deformed plate and color map of out-of-plane displacement obtained using A) ANSYS B) Present method, for a laminate plate made of 50 plies. Due to non-zero coupling matrix B, in-plane loads leads to out-of-plane bending.

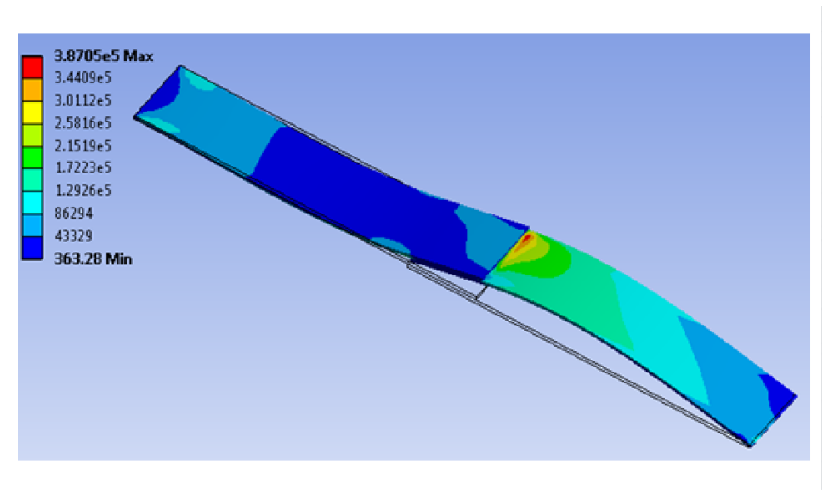

(A)

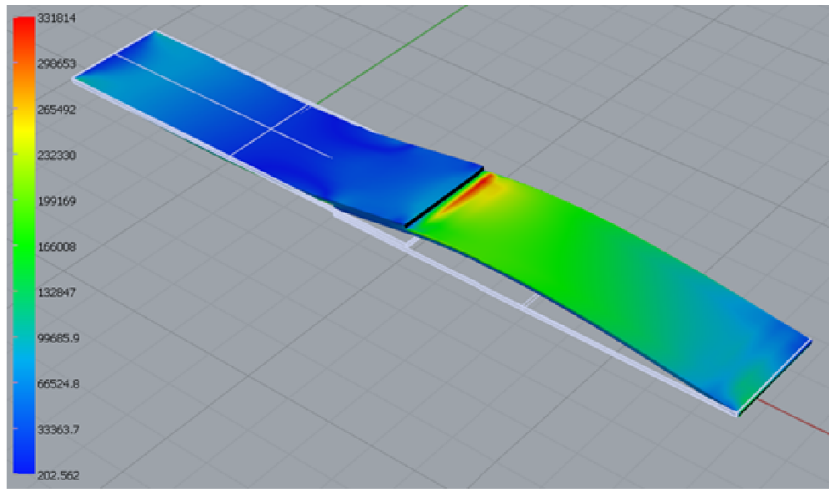

(B)

Figure 17: Figure shows color map of Von Mises stress for the lap joint made of 50 plies obtained using A) ANSYS (Max stress- $3.87 e^{5}$ psi) B) Present method (Max stress- $3.32 e^{5}$ psi). 

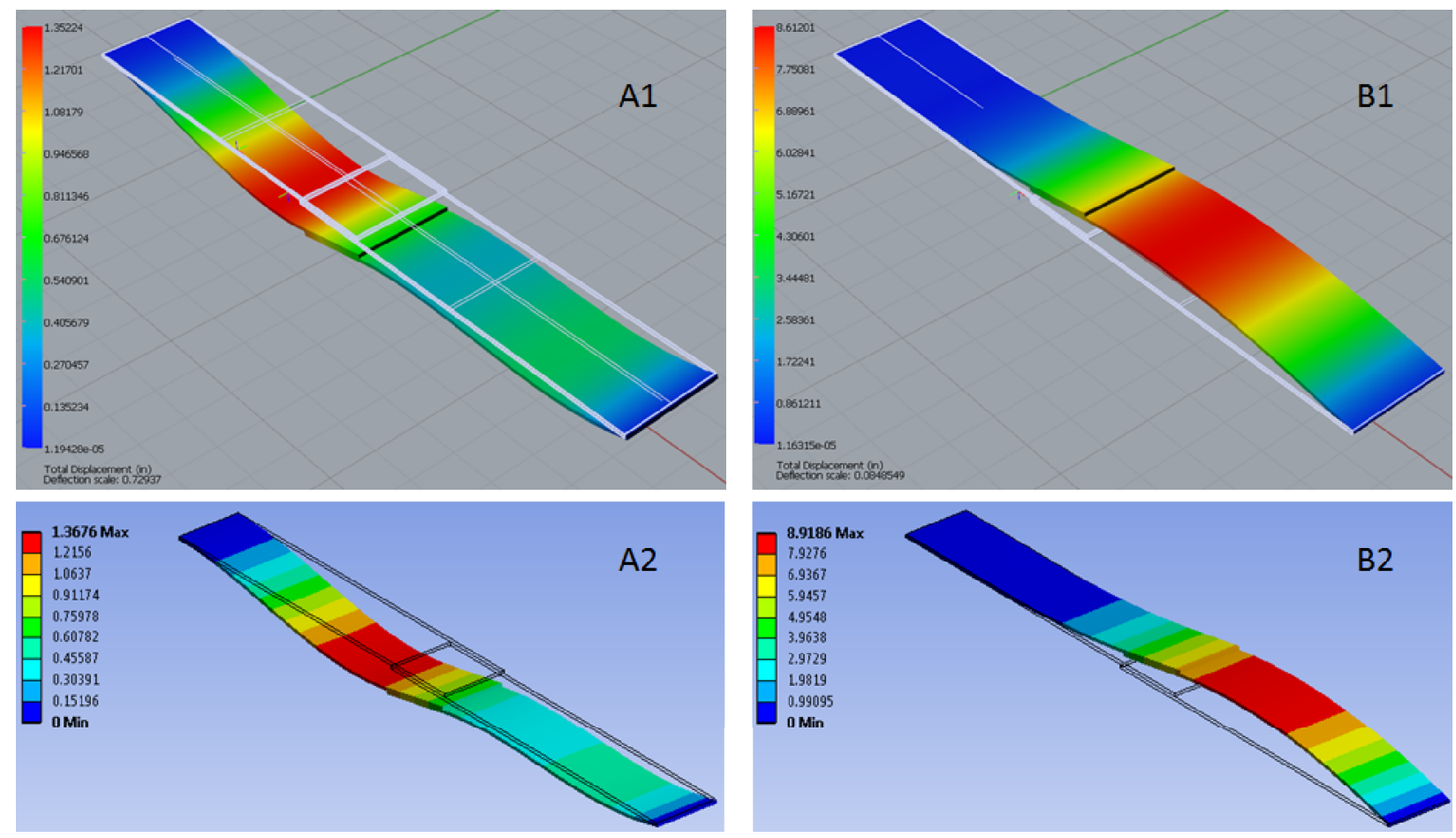

Figure 18: Figure A shows deformation in lap joint made of laminate [0,90] in 1) SnS, and 2) ANSYS when $0^{\circ}$ plies of the two laminates are bonded. Figure B shows deformation in the lap joint when $90^{\circ}$ plies of the two laminates are bonded.

predicted, the deformation in the lap joints obtained using proposed method and ANSYS agree.

Observe that the lap joints in Figures 18 and 19 exhibit significantly different deformation patterns, even though the geometry and the boundary conditions are identical. A plausible explanation is as follows. When stretching-bending coupling is not strong, bending due to eccentric forces in the lap joint dominates, and the deformed lap joint looks like Figure 16. However, for the lap joint made of [0/90] laminate in Figure 18A, stretching-bending coupling is strong and, in Laminate 2, the resultant moment is in the direction opposite to the direction of the moment due to eccentric forces. This causes Laminate 2 to bend in the opposite direction when compared to Figure 16 . On the other hand, for Laminate 2 of the lap joint in Figures 18B, bending due to stretching-bending coupling is in the same direction as bending due to the eccentric forces. Therefore, the out-of-plane deformation pattern is similar, but higher in value when compared to the deformation in Figure 16. For the lap joint made of $[-45,45]$ laminate in Figure 19, twisting moment is generated due to coupling in addition to the bending moment due to eccentric forces; therefore, the out-ofplane deformation is not uniform in the width, or $y$, direction. The direction of the twist reverses when the $-45^{\circ}$ plies are bonded together instead of the $45^{\circ}$ plies. 

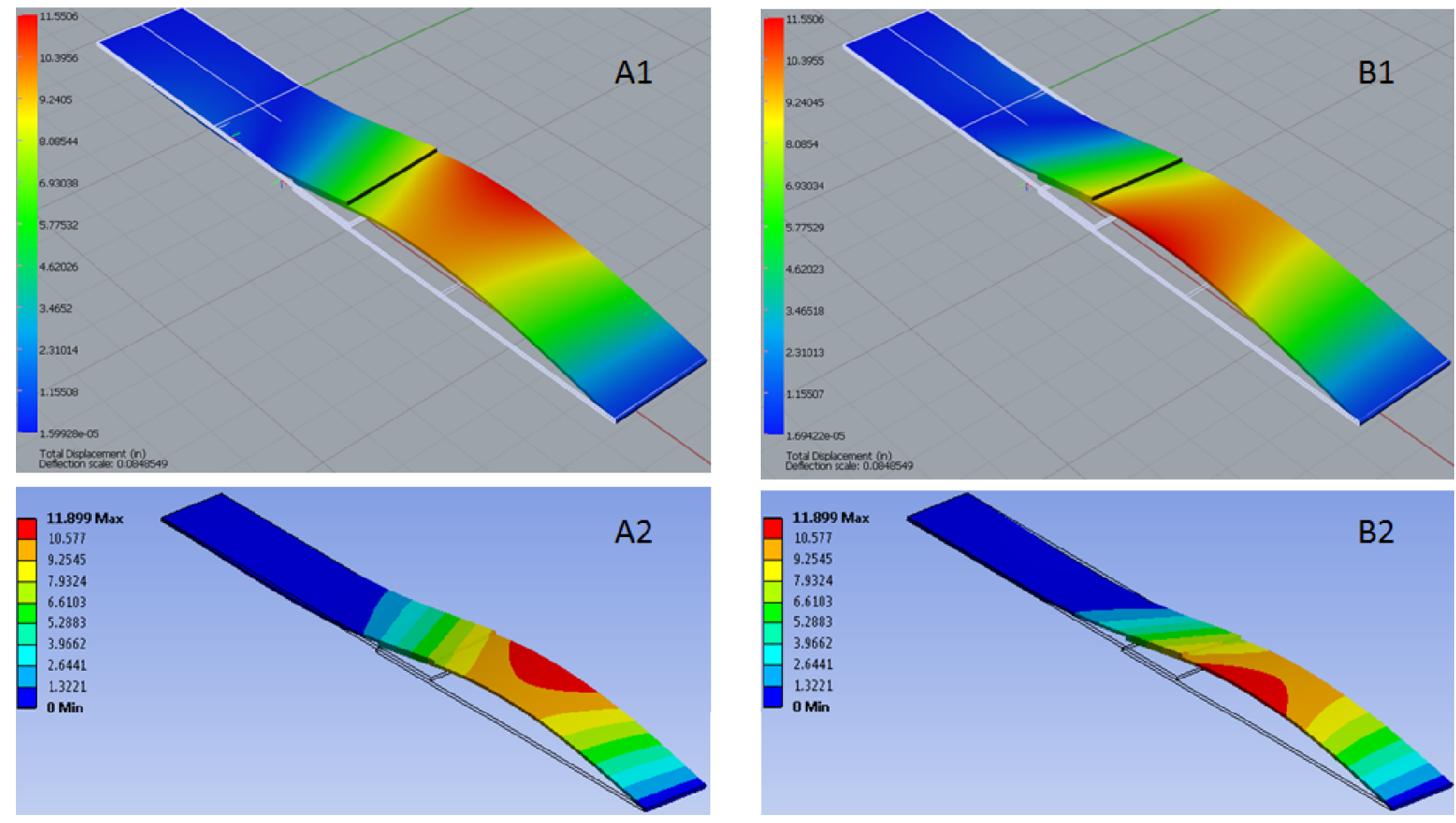

Figure 19: Figure A shows deformation in lap joint made of laminate $[-45,45]$ in 1) SnS, and 2) ANSYS when $45^{\circ}$ plies of the two laminates are bonded. Figure B shows deformation in the lap joint when $-45^{\circ}$ plies of the two laminates are bonded. 


\section{Conclusion and Future Work}

We proposed a new approach to modeling composite laminates that allows significant reduction in computational cost of 3D structural simulation. The essence of the approach is to replace the actual $n$-ply laminate structure with a virtual ABD-equivalent material model that behaves identically under the assumption of the lamination theory but is much simpler to analyze. In the current work, we examined two such models: a 3-ply model, and a quadratically varying graded material model. The complexity of these models remains constant and is independent of the complexity of the actual physical laminate. This implies that the computational efficiency gain grows linearly for 3D FEA: for an $n$-ply laminate structure, one can expect $O(n / 3)$ efficiency gain with the 3 -ply model and $O(3 n / 4)$ for the graded model using Lobatto quadrature rules for integration.

These ABD-equivalent models can be implemented in any 3D FEA system which supports laminates, effectively taking advantage of dimensional reduction while still using general 3D finite elements. We also demonstrated that with a suitable choice of basis functions, the ABD-equivalent models make 3D FEA practical and efficient enough to be considered an attractive alternative to existing semi-automated methods.

We validated the proposed ABD-equivalent material models by showing that their use in 3D meshfree analysis system leads to results that are consistently in good agreement with known results for several benchmark problems. In our numerical tests, we used the ABD-equivalent material model everywhere in the laminate structures, but in practice, ABDequivalent material models should be used only in plate/shell regions of the structures. Our future work will focus on incorporating such ABD-equivalent material models into general 3D analysis of complex composite structures with multiple laminates, joints, and discontinuities. The immediate challenges include identifying regions in the laminate where such models can be used, and efficient handling of the regions where they cannot be used. Finally, we note that equivalent materials may be also derived for higher order lamination theories [19] further improving the accuracy of 3D FEA with reduced computational cost.

\section{Acknowledgment}

This research was supported in part by National Science Foundation grants CMMI1344205, CMMI-1029553, and National Institute of Standards Research Contract SB134113-CN-0032. The authors are also grateful to Michael Freytag of Intact Solutions for providing programming interface and support for Scan\&Solve software. The responsibility for errors and omissions lie solely with the authors.

\section{Appendix}

\section{Appendix A. Constitutive Relationships}

\section{Appendix A.1. Full Constitutive Relationships for Orthotropic Plies}

For an orthotropic material, constitutive relationship relating stresses and strains in material principal direction is given as: 


$$
\left[\begin{array}{c}
\sigma_{x} \\
\sigma_{y} \\
\sigma_{z} \\
\tau_{y z} \\
\tau_{x z} \\
\tau_{x y}
\end{array}\right]=\left[\begin{array}{cccccc}
C_{11} & C_{12} & C_{13} & 0 & 0 & 0 \\
C_{12} & C_{22} & C_{23} & 0 & 0 & 0 \\
C_{13} & C_{23} & C_{33} & 0 & 0 & 0 \\
0 & 0 & 0 & C_{44} & 0 & 0 \\
0 & 0 & 0 & 0 & C_{55} & 0 \\
0 & 0 & 0 & 0 & 0 & C_{66}
\end{array}\right] \cdot\left[\begin{array}{c}
\epsilon_{x} \\
\epsilon_{y} \\
\epsilon_{z} \\
\gamma_{y z} \\
\gamma_{x z} \\
\gamma_{x y}
\end{array}\right]
$$

In a coordinate system different from material coordinate system, the constitutive matrix has to be transformed and is given as:

$$
\mathbf{C}^{\prime}=\mathbf{G}^{T} \cdot \mathbf{C} \cdot \mathbf{G}
$$

where $G$ is the transformation matrix. $G$ in terms of direction cosines of material directions $l, r, t$ with respect to general coordinate directions $x, y, z$ is given as:

$$
G=\left[\begin{array}{cccccc}
l_{x}^{2} & l_{y}^{2} & l_{z}^{2} & l_{y} l_{z} & l_{x} l_{z} & l_{x} l_{y} \\
r_{x}^{2} & r_{y}^{2} & r_{z}^{2} & r_{y} r_{z} & r_{x} r_{z} & r_{x} r_{y} \\
t_{x}^{2} & t_{y}^{2} & t_{z}^{2} & t_{y} t_{z} & t_{x} t_{z} & t_{x} t_{y} \\
2 r_{x} t_{x} & 2 r_{y} t_{y} & 2 r_{z} t_{z} & r_{y} t_{z}+r_{z} t_{y} & r_{z} t_{x}+r_{x} t_{z} & r_{x} t_{y}+r_{y} t_{x} \\
2 l_{x} t_{x} & 2 l_{y} t_{y} & 2 l_{z} t z & l_{y} t_{z}+l_{z} t_{y} & l_{z} t_{x}+l_{x} t_{z} & l_{x} t_{y}+l_{y} t_{x} \\
2 l_{x} r_{x} & 2 l_{y} r_{y} & 2 l_{z} r_{z} & l_{y} r_{z}+l_{z} r_{y} & l_{z} r_{x}+l_{x} r_{z} & l_{x} r_{y}+l_{y} r_{x}
\end{array}\right] .
$$

Appendix A.2. Plane-stress Constitutive Relationship for Orthotropic Materials

For thin structures, stresses in z direction are negligible, and plane-stress assumptions result in a $2 \mathrm{D}$ constitutive model, which in arbitrary coordinate system is given as:

$$
\left[\begin{array}{c}
\sigma_{x} \\
\sigma_{y} \\
\tau_{x y}
\end{array}\right]=\left[\begin{array}{lll}
Q_{11} & Q_{12} & Q_{13} \\
Q_{12} & Q_{22} & Q_{23} \\
Q_{13} & Q_{23} & Q_{33}
\end{array}\right] \cdot\left[\begin{array}{c}
\epsilon_{x} \\
\epsilon_{y} \\
\gamma_{x y}
\end{array}\right]
$$

\section{Appendix B. Classical Lamination Plate Theory}

When a structure is in stretching and pure bending, strains $\epsilon_{i}$ are assumed to vary linearly in the laminate's thickness direction. In terms of strains $\epsilon_{i}^{o}$ and curvatures $\kappa_{i}$ at the mid-plane, $\epsilon_{i}$ are given as:

$$
\epsilon_{i}=\epsilon_{i}^{o}+z \cdot \kappa_{i} \quad \mathrm{i}=1,2,3 .
$$

The stresses in the thickness direction of the structure can be integrated and reduced to forces and moments respectively [4]. Stresses summed along the thickness result in forces per unit length $N_{i}[4]$ :

$$
N_{i}=\int_{-\frac{t}{2}}^{\frac{t}{2}} \sigma_{i} d z=\int_{-\frac{t}{2}}^{\frac{t}{2}} Q_{i j} \cdot \epsilon_{j} d z=\int_{-\frac{t}{2}}^{\frac{t}{2}} Q_{i j}\left(\epsilon_{j}^{o}+z \cdot \kappa_{j}\right) d z
$$


Since $\epsilon^{o}$ and $\kappa$ are constant across thickness, above equation can be further reduced:

$$
N_{i}=\left(\int_{-\frac{t}{2}}^{\frac{t}{2}} Q_{i j} d z\right) \cdot \epsilon_{j}^{o}+\left(\int_{-\frac{t}{2}}^{\frac{t}{2}} Q_{i j} z d z\right) \cdot \kappa_{j}
$$

which can be rewritten in matrix forms as:

$$
\mathbf{N}=\mathbf{A} \cdot \epsilon^{o}+\mathbf{B} \cdot \kappa
$$

Using the fact that a laminate is made of plies (Figure 2), and material properties $Q_{i j}^{k}$ of the $k^{t h}$ ply is constant within that ply, matrices $\mathbf{A}$ and $\mathbf{B}$ can be further simplified:

$$
\begin{aligned}
A_{i j} & =\int_{-\frac{t}{2}}^{\frac{t}{2}} Q_{i j} d z=\sum_{k=1}^{n} \int_{h_{k}-1}^{h_{k}} Q_{i j}^{k} d z=\sum_{k=1}^{n} Q_{i j}^{k}\left(h_{k}-h_{k-1}\right), \\
\text { and } \quad B_{i j}=\int_{-\frac{t}{2}}^{\frac{t}{2}} Q_{i j} z d z & =\sum_{k=1}^{n} \int_{h_{k}-1}^{h_{k}} Q_{i j}^{k} z d z=\sum_{k=1}^{n} Q_{i j}^{k}\left(h_{k}^{2}-h_{k-1}^{2}\right),
\end{aligned}
$$

where $k^{\text {th }}$ ply lies between heights $h_{k-1}$ and $h_{k}$.

Similarly, resultant moments per unit length $M_{i}$ can also be obtained:

$$
M_{i}=\int_{-\frac{t}{2}}^{\frac{t}{2}} \sigma_{i} z d z=\int_{-\frac{t}{2}}^{\frac{t}{2}} Q_{i j} \cdot \epsilon_{j} z d z=\int_{-\frac{t}{2}}^{\frac{t}{2}} Q_{i j}\left(\epsilon_{j}^{o}+z \cdot \kappa_{j}\right) z d z
$$

Again, since $\epsilon^{o}$ and $\kappa$ are constant across the thickness, moments $M_{i}$ reduce to

$$
M_{i}=\left(\int_{-\frac{t}{2}}^{\frac{t}{2}} Q_{i j} z d z\right) \cdot \epsilon_{j}^{o}+\left(\int_{-\frac{t}{2}}^{\frac{t}{2}} Q_{i j} z^{2} d z\right) \cdot \kappa_{j}
$$

which, in matrix form, is given as:

$$
\mathbf{M}=\mathbf{B} \cdot \epsilon^{o}+\mathbf{D} \cdot \kappa
$$

Matrices $\mathbf{B}$ is same as above, while $\mathbf{D}$ is given as:

$$
D_{i j}=\int_{-\frac{t}{2}}^{\frac{t}{2}} Q_{i j} z^{2} d z=\sum_{k=1}^{n} \int_{h_{k}-1}^{h_{k}} Q_{i j}^{k} z^{2} d z=\sum_{k=1}^{n} Q_{i j}^{k}\left(h_{k}^{3}-h_{k-1}^{3}\right) .
$$

\section{Appendix C. Derivation of ABD-equivalent Models}

\section{Appendix C.1. 3-Ply Model}

The three plies have material properties $Q_{i j}^{* k}$ for $k=1,2,3$. Also assume the first ply lies between $h_{0}$ and $h_{1}$, the second between $h_{1}$ and $h_{2}$, and the third between $h_{2}$ and $h_{3}$. 
Since the 3-ply model must result in ABD matrices identical to the given ABD matrices, $Q_{i j}^{* k}$ will satisfy the following equations:

$$
\begin{array}{ccc}
A_{i j}^{o} & =\quad \sum_{k=1}^{3} \int_{h_{k}-1}^{h_{k}} Q_{i j}^{* k} d z=Q_{i j}^{* 1}\left(h_{1}-h_{0}\right)+Q_{i j}^{* 2}\left(h_{2}-h_{1}\right)+Q_{i j}^{* 3}\left(h_{3}-h_{2}\right), \\
B_{i j}^{o}= & \sum_{k=1}^{3} \int_{h_{k}-1}^{h_{k}} Q_{i j}^{* k} z d z=\frac{1}{2}\left\{Q_{i j}^{* 1}\left(h_{1}^{2}-h_{0}^{2}\right)+Q_{i j}^{* 2}\left(h_{2}^{2}-h_{1}^{2}\right)+Q_{i j}^{* 3}\left(h_{3}^{2}-h_{2}^{2}\right)\right\} \\
& =c \\
D_{i j}^{o} & =\sum_{k=1}^{3} \int_{h_{k}-1}^{h_{k}} Q_{i j}^{* k} z^{2} d z=\frac{1}{3}\left\{Q_{i j}^{* 1}\left(h_{1}^{2}-h_{0}^{2}\right)+Q_{i j}^{* 3}\left(h_{3}^{2}-h_{2}^{2}\right)\right\},
\end{array}
$$

Assuming that the three plies are of equal thickness, and the total laminate thickness is $t$, above equations can be solved for $Q_{i j}^{* k}$ and is given in Equation 12.

\section{Appendix C.2. Graded Material Model}

The quadratically graded material $Q_{i j}^{*}=z^{2} \Lambda_{i j}^{2}+z \Lambda_{i j}^{1}+\Lambda_{i j}^{0}$ also must result in ABD matrices identical to the given $\mathrm{ABD}$ matrices. Therefore, $Q_{i j}^{*}$ must satisfy the following equations:

$$
\begin{aligned}
A_{i j}^{o} & =\quad \int_{-\frac{t}{2}}^{\frac{t}{2}} Q_{i j}^{*} d z=\int_{-\frac{t}{2}}^{\frac{t}{2}}\left(z^{2} \Lambda_{i j}^{2}+z \Lambda_{i j}^{1}+\Lambda_{i j}^{0}\right) d z \\
& =\quad\left[\frac{z^{3}}{3} \Lambda_{i j}^{2}+\frac{z^{2}}{2} \Lambda_{i j}^{1}+z \Lambda_{i j}^{0}\right]_{-\frac{t}{2}}^{\frac{t}{2}}=\frac{t^{3}}{12} \Lambda_{i j}^{2}+t \Lambda_{i j}^{0}, \\
B_{i j}^{o} & =\quad \int_{-\frac{t}{2}}^{\frac{t}{2}} Q_{i j}^{*} z d z=\int_{-\frac{t}{2}}^{\frac{t}{2}}\left(z^{2} \Lambda_{i j}^{2}+z \Lambda_{i j}^{1}+\Lambda_{i j}^{0}\right) z d z \\
& =\quad\left[\frac{z^{4}}{4} \Lambda_{i j}^{2}+\frac{z^{3}}{3} \Lambda_{i j}^{1}+\frac{z^{2}}{2} \Lambda_{i j}^{0}\right]_{-\frac{t}{2}}^{\frac{t}{2}}=\frac{t^{3}}{12} \Lambda_{i j}^{1}, \\
D_{i j}^{o} & =\int_{-\frac{t}{2}}^{\frac{t}{2}} Q_{i j}^{*} z^{2} d z=\int_{-\frac{t}{2}}^{\frac{t}{2}}\left(z^{2} \Lambda_{i j}^{2}+z \Lambda_{i j}^{1}+\Lambda_{i j}^{0}\right) z^{2} d z \\
& =\left[\frac{z^{5}}{5} \Lambda_{i j}^{2}+\frac{z^{4}}{4} \Lambda_{i j}^{1}+\frac{z^{3}}{3} \Lambda_{i j}^{0}\right]_{-\frac{t}{2}}^{\frac{t}{2}}=\frac{t^{5}}{80} \Lambda_{i j}^{2}+\frac{t^{3}}{12} \Lambda_{i j}^{0} .
\end{aligned}
$$

Above equations can be solved for $\Lambda_{i j}^{k}$ for $k=1,2,3$ and is given in Equation 14 .

\section{Appendix D. Laminate with 50 Random Plies}

Laminate with 50 random plies has the following fiber orientations: $-45,45,0,-45,90$, $45,-50,-75,60,-45,90,-45,-45,45,-45,-75,-5,80,30,-45,-45,60,90,-75,-45,25,-45$, $-45,45,-75,60,60,-45,90,-45,-45,-75,-50,45,-45,60,-45,50,-75,-45,-75,10,-45,60$, and 90 .

\section{References}

[1] R. F. Gibson, Principles of composite material mechanics, CRC Press, 2011.

[2] F. C. Campbell Jr, Manufacturing processes for advanced composites, Access Online via Elsevier, 2003.

[3] A. Handbook, Vol. 21, Composites 380. 
[4] I. M. Daniel, O. Ishai, I. M. Daniel, I. Daniel, Engineering mechanics of composite materials, Vol. 3, Oxford university press New York, 1994.

[5] J. N. Reddy, Mechanics of Laminated Composite Plates and Shells: Theory and Analysis/JN Reddy, CRC press, 2004.

[6] F. L. Matthews, Finite element modelling of composite materials and structures, CRC Press, 2000.

[7] K. J. Saeger, P. A. Lagace, D. J. Shim, Interlaminar stresses due to in-plane gradient stress fields, Journal of composite materials 36 (2) (2002) 211-227.

[8] J. Reddy, An evaluation of equivalent-single-layer and layerwise theories of composite laminates, Composite Structures 25 (1) (1993) 21-35.

[9] R. D. Cook, et al., Concepts and applications of finite element analysis, Wiley. com, 2007.

[10] S. Klinkel, F. Gruttmann, W. Wagner, A continuum based three-dimensional shell element for laminated structures, Computers \& Structures 71 (1) (1999) 43-62.

[11] K. Dorninger, F. G. Rammerstorfer, A layered composite shell element for elastic and thermoelastic stress and stability analysis at large deformations, International Journal for Numerical Methods in Engineering 30 (4) (1990) 833-858.

[12] M. Chatiri, T. Güll, A. Matzenmiller, An assessment of the new ls-dyna layered solid element: basics, patch simulation and its potential for thick composite structure analysis, in: 7th European LS-DYNA Conference, Salzburg, 2009.

[13] J. O. Dow, D. E. Byrd, The identification and elimination of artificial stiffening errors in finite elements, International Journal for Numerical Methods in Engineering 26 (3) (1988) 743-762.

[14] A. Düster, H. Bröker, E. Rank, The p-version of the finite element method for three-dimensional curved thin walled structures, International Journal for Numerical Methods in Engineering 52 (7) (2001) 673703.

[15] M. Suri, Analytical and computational assessment of locking in thei i $\mathrm{ip}$ i/ii, finite element method, Computer Methods in Applied Mechanics and Engineering 133 (3) (1996) 347-371.

[16] Y. Zhang, C. Yang, Recent developments in finite element analysis for laminated composite plates, Composite Structures 88 (1) (2009) 147-157.

[17] T. Kant, K. Swaminathan, Estimation of transverse/interlaminar stresses in laminated composites-a selective review and survey of current developments, Composite structures 49 (1) (2000) 65-75.

[18] E. Carrera, Theories and finite elements for multilayered, anisotropic, composite plates and shells, Archives of Computational Methods in Engineering 9 (2) (2002) 87-140.

[19] H. Matsunaga, Assessment of a global higher-order deformation theory for laminated composite and sandwich plates, Composite Structures 56 (3) (2002) 279-291.

[20] L. J. Hart-Smith, Adhesive-bonded single-lap joints, Langley Research Center Hampton, VA, 1973.

[21] G. Kumar, V. Shapiro, Analysis of multi-material bonded assemblies on a non-conforming mesh, in: ASME 2012 International Design Engineering Technical Conferences and Computers and Information in Engineering Conference, American Society of Mechanical Engineers, 2012, pp. 111-123.

[22] K. Suresh, Automating the cad/cae dimensional reduction process, in: Proceedings of the eighth ACM symposium on Solid modeling and applications, ACM, 2003, pp. 76-85.

[23] O. C. Zienkiewicz, R. L. Taylor, The finite element method for solid and structural mechanics, Butterworth-Heinemann, 2005.

[24] K.-J. Bathe, E. N. Dvorkin, A formulation of general shell elementsthe use of mixed interpolation of tensorial components, International Journal for Numerical Methods in Engineering 22 (3) (1986) 697-722.

[25] S. Hossain, P. Sinha, A. H. Sheikh, A finite element formulation for the analysis of laminated composite shells, Computers \& structures 82 (20) (2004) 1623-1638.

[26] M. Freytag, V. Shapiro, I. Tsukanov, Finite element analysis in situ, Finite Elements in Analysis and Design 47 (9) (2011) 957-972.

[27] A. Idlbi, M. Karama, M. Touratier, Comparison of various laminated plate theories, Composite Structures 37 (2) (1997) 173-184.

[28] C. Sun, S. Li, Three-dimensional effective elastic constants for thick laminates, Journal of Composite 
Materials 22 (7) (1988) 629-639.

[29] ASME, Asme y14.37-2012 composite part drawings.

[30] A. R. Krommer, Numerical Integration: On Advanced Computer Systems, Vol. 848, Springer, 1994.

[31] I. SolidWorks, Solidworks corporation, Concord, MA.

[32] A. U. Manual, Ansys inc, Canonsburg, PA. 